\title{
How to Tell Ancient Signs Apart? Recognizing and Visualizing Maya Glyphs with CNNs
}

GÜLCAN CAN, Idiap Research Institute and École Polytechnique Fédérale de Lausanne (EPFL), Switzerland JEAN-MARC ODOBEZ, Idiap Research Institute and École Polytechnique Fédérale de Lausanne (EPFL), Switzerland

DANIEL GATICA-PEREZ, Idiap Research Institute and École Polytechnique Fédérale de Lausanne (EPFL), Switzerland

Thanks to the digital preservation of cultural heritage material, multimedia tools, e.g. based on automatic visual processing, enable to considerably ease the work of scholars in the humanities and help them to perform quantitative analysis of their data. In this context, this paper assesses three different Convolutional Neural Network (CNN) architectures along with three learning approaches to train them for hieroglyph classification, which is a very challenging task due to the limited availability of segmented ancient Maya glyphs. More precisely, the first approach, the baseline, relies on pretrained networks as feature extractor. The second one investigates a transfer learning method by fine-tuning a pretrained network for our glyph classification task. The third approach considers directly training networks from scratch with our glyph data. The merits of three different network architectures are compared: a generic sequential model (i.e. LeNet), a sketch-specific sequential network (i.e. Sketch-a-Net), and the recent Residual Networks. The sketch-specific model trained from scratch outperforms other models and training strategies. Even for a challenging 150-class classification task, this model achieves $70.3 \%$ average accuracy and proves itself promising in case of small amount of cultural heritage shape data. Furthermore, we visualize the discriminative parts of glyphs with the recent Grad-CAM method, and demonstrate that the discriminative parts learned by the model agrees in general with the expert annotation of the glyph specificity (diagnostic features). Finally, as a step towards systematic evaluation of these visualizations, we conduct a perceptual crowdsourcing study. Specifically, we analyze the interpretability of the representations from Sketch-a-Net and ResNet-50. Overall, our paper takes two important steps towards providing tools to scholars in the digital humanities: increased performance for automation, and improved interpretability of algorithms.

CCS Concepts: • Computing methodologies $\rightarrow$ Shape representations; Neural networks; Object identification; • Applied computing $\rightarrow$ Arts and humanities;

Additional Key Words and Phrases: Maya glyphs, shape recognition, convolutional neural networks, transfer learning, crowdsourcing

ACM Reference Format:

Gülcan Can, Jean-Marc Odobez, and Daniel Gatica-Perez. 2018. How to Tell Ancient Signs Apart? Recognizing and Visualizing Maya Glyphs with CNNs. ACM F. Comput. Cult. Herit. 9, 4, Article 1 (June 2018), 25 pages. https://doi.org/0000001.0000001

This work was funded by the Swiss National Science Foundation through the MAAYA project and by the Hasler Foundation through the DCrowdLens project. Authors' addresses: G. Can, J.M. Odobez, and D. Gatica-Perez are with Idiap Research Institute, and the School of Electrical Engineering of the Swiss Federal Institute of Technology in Lausanne (EPFL), Switzerland. e-mail: gcan@idiap.ch, odobez@idiap.ch, and gatica@idiap.ch.

Permission to make digital or hard copies of all or part of this work for personal or classroom use is granted without fee provided that copies are not made or distributed for profit or commercial advantage and that copies bear this notice and the full citation on the first page. Copyrights for components of this work owned by others than the author(s) must be honored. Abstracting with credit is permitted. To copy otherwise, or republish, to post on servers or to redistribute to lists, requires prior specific permission and/or a fee. Request permissions from permissions@acm.org.

(c) 2018 Copyright held by the owner/author(s). Publication rights licensed to Association for Computing Machinery.

XXXX-XXXX/2018/6-ART1 $\$ 15.00$

https://doi.org/0000001.0000001

ACM J. Comput. Cult. Herit., Vol. 9, No. 4, Article 1. Publication date: June 2018. 


\section{INTRODUCTION}

This paper focuses on learning shape representations for ancient Maya hieroglyph classification task. In document analysis of historical and artistic materials, visual similarity-based recognition is important for helping experts to assess shapes quantitatively and to annotate documents easily. Encoding shapes into discriminative representations is essential for visual similarity tasks. As traditional shape descriptors, e.g. [24] have limits for degraded characters in old manuscripts (see Fig. 1) or sparse shapes such as sketches, learning robust shape representations from data is of great interest to multimedia research.

In parallel, recent advancements with deep Convolutional Neural Networks (CNNs) for recognition tasks in computer vision, speech, and multimedia have proven the usefulness of these methods [22]. However, applying such techniques when dealing with small amounts of data is challenging. In this paper, as the first research question, we study how to utilize CNNs for Maya glyph classification. By relying on the data of the three remaining Maya Codices produced in our recent study [3], we investigate three different strategies to learn such a representation: using a pretrained $\mathrm{CNN}$ as a feature extractor, doing transfer learning (i.e. fine-tuning) from a pretrained $\mathrm{CNN}$, and learning a $\mathrm{CNN}$ from scratch.

Deep representations are shown as strong baselines for various visual recognition tasks [6, 9, 35, 37]. The second research question addressed in this paper is which deep CNN architecture to choose for Maya glyph classification. Assuming that Maya shapes painted on codices share commonalities with hand-drawn sketches and everyday objects in natural images, first, we evaluate the deep representations learned on natural images and on sketches as baseline methods for our task. Specifically, we study Sketch-a-Net [43] pretrained on Sketch-250 dataset [10], VGG-16 [37] and ResNet-50 [13] pretrained on ImageNet dataset [7]. Secondly, we investigate transferring the knowledge learned on a large-scale dataset to our specific problem by fine-tuning a pretrained $\mathrm{CNN}$ as in [42]. This approach was shown to be useful in a variety of multimedia applications $[1,6,40]$, and is particularly valuable when the data collection is not scalable, either due to the involved cost and time, ethical and security reasons (i.e. medical data), or simply because of extinct data sources (i.e. scripts from the ancient civilizations). We show that in spite of the larger discrepancy between the source (ImageNet) and target (glyph) data, VGG-16 outperforms Sketch-a-Net by $8.9 \%$ to $14.8 \%$ in average classification accuracy. In particular, we show 2.4 to $14.4 \%$ absolute performance improvement by fine-tuning the VGG-16 model, compared to the feature extraction baseline results. Thirdly, we train CNNs from scratch with the glyph data. Usually, training a CNN from scratch requires a large amount of data. However, recent work on batch normalization (BN) [19] and dropout regularization [15], along with oversampling via data augmentation (i.e. applying random geometric transformations to the data) enable training a $\mathrm{CNN}$ for small- to medium-scale data while reducing data imbalance
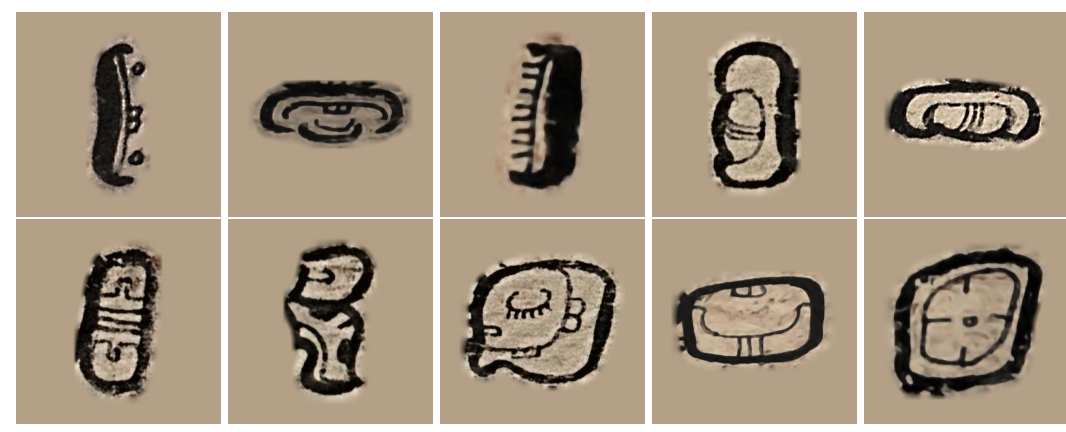

Fig. 1. Examples of ancient Maya codical glyphs (10 classes). 


$$
\begin{array}{r}
\text { How to Tell Ancient Signs Apart? } \\
\text { Recognizing and Visualizing Maya Glyphs with CNNs - } 1: 3
\end{array}
$$

[14] and overfitting issues. This way, we achieve improved results with Sketch-a-Net compared to the fine-tuning approach, demonstrating that nowadays network training may not necessarily require large amount of data to be effective.

The third research question is posed as whether the CNNs are able to catch the diagnostic parts of Maya glyphs. To analyze the interpretability of the learned deep visual representations, we visualize the discriminative parts of glyphs according to the trained CNNs via gradient backpropagation [36] and Grad-CAM [34] methods. We show that the trained models have a great interpretability potential, as the discriminative parts of glyphs overlap with the expert descriptions in a 5-glyph case study. Additionally, we discuss the potential of Grad-CAM method in glyph localization in a cluttered setting, i.e. glyph-blocks. Finally, to assess the GradCAM visualizations thoroughly, we conduct a perceptual crowdsourcing study. The fourth research question assessed in this study is which CNN spots diagnostic parts of glyphs better according to non-experts. In this study, non-experts rate the visualizations from two models, namely Sketch-a-Net and ResNet-50, both individually and relatively for 100 glyphs. From these ratings, we observe that non-experts generally favor the better-focused visualizations from Sketch-a-Net.

We summarize our research questions below.

RQ1. How to utilize deep CNNs on Maya glyph classification?

RQ2. Which deep CNN architecture to choose for Maya glyph classification?

RQ3. Can CNNs catch diagnostic parts of Maya glyphs?

RQ4. If so, which CNN spots diagnostic parts of glyphs better according to the crowdworkers' perception?

Our contributions are as follows:

(1) We study different ways to train a CNN for small-scale data, namely feature extraction from pretrained networks, fine-tuning pretrained networks, and training from scratch for the recognition of ancient Maya glyphs, a data source between history and art;

(2) We systematically assess a variety of CNN models from old to recent ones, i.e. LeNet [23], Sketch-a-Net [43], VGG [37], ResNet [13], and designed for different purposes (natural image vs. sketch classification);

(3) We examine the discriminative parts of ancient glyphs learned by the network using gradient-based visual explanations. This can provide an interesting interpretability capacity, which is important for domain experts.

(4) We conduct a perceptual crowdsourcing study to assess the interpretability capacity of two models, namely Sketch-a-Net and ResNet, by the help of GradCAM [34] visualization method.

Overall, our paper presents promising shape representation learning approaches for limited cultural heritage data as well as giving insights on the interpretability of these representations. These two points are important in the context of providing automated and interpretable tools to scholars in Digital Humanities.

The paper is organized as follows. Section 2 briefly presents related works. Section 3 describes the data. Section 4 summarizes the methodology. Section 5 presents the classification results (RQ1 \& RQ2). Section 5.4 discusses the interpretability of the trained CNN models (RQ3), and presents our perceptual crowdsourcing study (RQ4). Finally, Section 6 concludes the paper.

\section{RELATED WORK}

This section discusses common architectures and training strategies with CNNs (i.e. analysis of pretrained CNN features, fine-tuning or training deep CNNs from scratch); previous glyph and sketch recognition studies; and visualization and interpretation of the learned representations.

CNN architectures. For handwritten digit classification, LeCun et al. proposed a sequential, i.e. feed-forward, network (LeNet) with three convolutional layers followed by a nonlinearity activation (e.g. sigmoid), and subsampling (e.g. max pooling) [23]. Krizhevsky et al. built upon the LeNet architecture, and proposed the AlexNet 
[21] that has five convolutional layers with non-saturating rectified linear unit (ReLU) activations for object classification on ImageNet data [7]. Vanishing/exploding gradients during error backpropagation through this deep net were handled by the ReLU activations. Additionally, the dropout strategy [15] helped to prevent overfitting during training of the AlexNet. VGG networks illustrated the limits of deep sequential networks without any special design [37]. Simonyan et al. showed that such a network with 16 layers (VGG-16) outperforms the AlexNet.

More recently, graph-based architectures have emerged in CNN design such as GoogleNet with inception modules [39], and residual networks (ResNets) with identity mapping connections [13]. One important commonality of these models is heavy-usage of Batch Normalization (BN) [19] which enables to train very deep networks in a considerably short amount of time with improved performance, since BN reduces covariate shift in the data during training.

Transfer learning and training with CNNs. Motivated by the common visual structures learned by CNNs in the first layers, several transfer learning approaches reutilized and analyzed the effectiveness of pretrained CNN representations on different datasets $[6,9,35,37]$. The penultimate activations of a CNN, specifically AlexNet [9,35] and VGG [37] trained on ImageNet data, are shown as strong baselines for visual recognition tasks. Similarly, for character recognition tasks e.g. uppercase Latin letter classification, Cireşan et al. [6] indicated that existing pretrained nets (trained on digits or Chinese characters) can be utilized as feature extractors.

Alternatively, these pretrained networks can be fine-tuned, i.e. trained such that only the weights of last several layers are modified, to be adapted to different tasks. Fine-tuning helps the training process start from a more relevant point and results in improved performance and faster training compared to random initialization [6]. Cireşan et al. [6] showed that even for the challenging task of 1000 Chinese character classification, fine-tuning the last convolutional and fully-connected layers of a pretrained net for uppercase Latin letters outperforms a randomly initialized and trained network ( $8.4 \%$ vs. $20.8 \%$ test error rate respectively). Furthermore, Yosinski et al. [42] showed that fine-tuning the last convolutional layers helps the network to learn representations that are more specific to the target dataset. Authors discuss that fine-tuning more number of layers (from last fully-connected layer towards input layer) might be essential as the nature of the target dataset becomes more different than the initial source dataset used for pretraining.

Training a CNN from scratch is one of the typical approaches in case of dissimilar data with particular characteristics, e.g. our glyph data. Even though the impact of pretraining is emphasized above, there are recent normalization techniques that reduce or eliminate the necessity of pretraining. Batch Normalization (BN) [19] is one of the most common approaches. BN showed considerable performance and efficiency improvements in CNN training [19]. Thus, when training a CNN from scratch in this paper, we initialized the network weights with Glorot initialization [12], and utilized BN after each convolutional layer.

Glyph and sketch recognition. For Maya glyph recognition, several shape representations have built upon traditional knowledge-driven descriptors [18, 30]. These representations are based on bag-of-words (BoW) that output the frequency histograms of local shape descriptors. As shown in a similar study on Egyptian glyphs [11], HOOSC [30] is a competitive candidate among other traditional shape descriptors.

Petroglyph, in other words rock-art, analysis $[8,28,31-33,46]$ is another related topic to our glyph recognition task. For petroglyph segmentation that can be considered as foreground/background classification of pixels, Seidl et al. [31] studied various combinations of traditional textural features. Specifically, third order Gray-Level Co-occurrence Matrix (GLCM) and Local Binary Patterns (LBP) were shown to outperform color or dense-SIFT features in a late classification fusion setting [31]. In a recent 3D petroglyph segmentation study, Poier et al. [28] reported that fully-connected CNNs produced better segmentation results thanks to capturing the spatial context better than random forests. Poier et al. [28] also noted that the contribution of traditional color and textural features to final segmentation maps was negligible, therefore only depth maps and orthophotos generated from the point clouds were used as input to the classifiers. 
How to Tell Ancient Signs Apart? Recognizing and Visualizing Maya Glyphs with CNNs • 1:5

Table 1. Comparison of contributions in the related work for glyph and sketch recognition.

\begin{tabular}{|c|c|c|c|c|}
\hline Paper & Task & Method & Dataset & $\begin{array}{l}\text { Visual } \\
\text { Interpretation }\end{array}$ \\
\hline$[30]$ & $\begin{array}{l}\text { Monumental } \\
\text { Maya glyph } \\
\text { retrieval }\end{array}$ & $\begin{array}{l}\text { HOOSC with } \\
\text { bag-of-words }\end{array}$ & $\begin{array}{l}\text { 24-class monumental } \\
\text { Maya glyph dataset [30] }\end{array}$ & No \\
\hline$[18]$ & $\begin{array}{l}\text { Monumental } \\
\text { Maya glyph } \\
\text { classification }\end{array}$ & HOOSC & $\begin{array}{l}\text { 10-class monumental } \\
\text { Maya glyphs } \\
\text { (subset of }[30] \text { ) }\end{array}$ & No \\
\hline [11] & $\begin{array}{l}\text { Egyptian } \\
\text { glyph } \\
\text { classification }\end{array}$ & $\begin{array}{l}\text { HOG, HOOSC, HOOSS, } \\
\text { Shape Context, Self-Similarity } \\
\text { (with bag-of-words or spatial } \\
\text { or single matching) }\end{array}$ & $\begin{array}{l}\text { Egyptian } \\
\text { individual } \\
\text { glyphs [11] }\end{array}$ & No \\
\hline [2] & $\begin{array}{l}\text { Monumental } \\
\text { Maya glyph } \\
\text { classification }\end{array}$ & HOOSC with bag-of-words & $\begin{array}{l}\text { 10-class monumental } \\
\text { Maya glyphs } \\
\text { (subset of }[30] \text { ) }\end{array}$ & No \\
\hline [2] & $\begin{array}{l}\text { Sketch } \\
\text { classification }\end{array}$ & $\begin{array}{l}\text { Single-hidden-layer } \\
\text { Sparse Autoencoders }\end{array}$ & $\begin{array}{l}\text { 250-class } \\
\text { sketch dataset [10] }\end{array}$ & No \\
\hline [43] & $\begin{array}{l}\text { Sketch } \\
\text { classification }\end{array}$ & $\begin{array}{l}\text { Ensemble of five } \\
\text { multi-scale } \\
\text { Sketch-a-Nets }\end{array}$ & $\begin{array}{l}250 \text {-class } \\
\text { sketch dataset [10] }\end{array}$ & No \\
\hline$[29]$ & $\begin{array}{l}\text { Codical Maya } \\
\text { glyph-block } \\
\text { retrieval }\end{array}$ & $\begin{array}{l}\text { HOOSC with bag of words, } \\
\text { pretrained features } \\
\text { from VGG network }\end{array}$ & $\begin{array}{l}\text { Codical Maya } \\
\text { glyph-blocks }\end{array}$ & No \\
\hline [3] & $\begin{array}{l}\text { Codical Maya } \\
\text { glyph } \\
\text { classification }\end{array}$ & $\begin{array}{l}\text { HOG, } \\
\text { HOOSC with bag of words, } \\
\text { pretrained features from } \\
\text { Sketch-a-Net, VGG-16, ResNet-50 } \\
\text { with original set of samples }\end{array}$ & $\begin{array}{l}\text { Codical Maya } \\
\text { glyphs [3] }\end{array}$ & No \\
\hline [4] & $\begin{array}{l}\text { Codical Maya } \\
\text { glyph } \\
\text { classification }\end{array}$ & $\begin{array}{l}\text { HOOSC with bag of words, } \\
\text { pretrained features from } \\
\text { Sketch-a-Net and VGG-16, } \\
\text { training Sketch-a-Net from scratch } \\
\text { with original set of samples } \\
\text { by applying class weights into loss function }\end{array}$ & $\begin{array}{l}\text { Codical Maya } \\
\text { glyphs [3] }\end{array}$ & No \\
\hline This paper & $\begin{array}{l}\text { Codical Maya } \\
\text { glyph } \\
\text { classification }\end{array}$ & $\begin{array}{l}\text { Pretrained features from } \\
\text { Sketch-a-Net, VGG-16, and ResNet-50 } \\
\text { finetuning the last convolutional block } \\
\text { of Sketch-a-Net, VGG-16, and ResNet-50, } \\
\text { training LeNet, Sketch-a-Net, } \\
\text { ResNet-18, and ResNet-50 from scratch } \\
\text { with oversampled balanced set of samples }\end{array}$ & $\begin{array}{l}\text { Codical Maya } \\
\text { glyphs [3] }\end{array}$ & Yes \\
\hline
\end{tabular}

In the context of Maya glyph classification, for encoding shapes with neural networks, a single-layer sparse autoencoder (SA), which encodes the same local regions as HOOSC, was shown to be competitive for 10-class monumental glyphs [2]. However, this shallow representation was not representative enough for other tasks, i.e. sketch classification task proposed in [10]. Due to the scarcity of the strokes in thin sketch drawings and the high variety of the drawings, the BoW frequencies of the simple edge encodings in the shallow SA were 
harder to capture than relatively-thicker glyph strokes. The authors of [2] discuss that deeper convolutional networks trained with more data might capture more complex and more discriminative shape representations for this case. Complementary to this finding, Yu et al.[43] proposed "Sketch-a-Net" that is a modified version of AlexNet [21] with fewer feature maps, yet larger first layer convolution kernels. Yu et al. demonstrated that a joint classification decision from a set of Sketch-a-Nets (trained with data in multiple scales and multiple temporal channels) beats human performance on the 250-class sketch dataset of [10].

In the context of Maya glyph-block retrieval, Roman-Rangel et al. [29] showed that the middle layer activations (conv5) of VGG outperform the penultimate layer activations (fc-7), and the bag-of-words representation of a traditional shape descriptor (HOOSC). This is a motivating point for learning the representations for Maya glyphs, and taking advantage from existing pretrained networks. Similarly, in our recent study [3], we presented the conv-5 activations of VGG-16 as a promising shape representation baseline on the challenging Maya Codical glyph dataset. In a related study [4], we evaluated the HOOSC-BoW representation along with the activations of two pretrained networks, namely VGG-16 and Sketch-a-Net, on this Maya Codical glyph dataset. The data-driven pretrained $\mathrm{CNN}$ representations outperformed the knowledge-driven HOOSC-BoW representation by a large margin. Furthermore, we trained a Sketch-a-Net from scratch with glyphs, and demonstrated that average class accuracies gave insights on the training process. We applied class weights in the loss function for the CNN not to overfit to the classes with many samples during training. This approach resulted in slight improvement in average class accuracies. Finally, to improve the performance of classes with few samples, we hinted that using oversampled balanced sets were essential.

In this paper, we substantially extend our work in [4] on the oversampled balanced sets of the Maya Codical glyph dataset. Specifically, we perform a more comprehensive study with different networks including the recent Residual Networks. Furthermore, we also experiment with another transfer learning approach by fine-tuning the pretrained networks. Additionally, we assess if the representations learned by the networks are indeed discriminative and interpretable by visualizing the network activations.

Interpreting CNN representations. To understand the representations learned by CNNs, Zeiler et al. discuss how to visualize them via deconvolutional layers [44]. They also present a method called occlusion maps such that a sliding window in the image is occluded and the predicted label of the image by the CNN model is checked to see whether that region is diagnostic and important to identify the correct label.

Simonyan et al. presented a simple gradient backpropagation approach for identifying the salient points of the objects with a single forward pass [36]. Compared to occlusion maps, it is computationally more efficient. However, this approach does not point out to full object extent in general. Therefore, the authors use the output salient points from this approach as input to a classical background/foreground segmentation method for object segmentation in natural images. In our case, the segmentation of glyphs from a glyph-block with classical approaches is especially challenging, since neither color nor texture are discriminative for glyphs.

Zhou et al. introduced class activation maps (CAM) [45] for capturing the extensions of objects and not only few salient points of objects. The CAM approach requires to introduce an average pooling layer to model structure. To avoid that, Grad-CAM [34] has been proposed as a generalization of CAM. As such, it does not require modifying the CNN model to visualize the activation maps, and it can be applied to any type of neural networks, even to pretrained ones without the need of re-training.

Beyond classification, interpretability is fundamental for domain experts, who need to understand what the method does and match it with their own knowledge. Therefore, we adopt the Grad-CAM approach for illustrating the discriminative parts of the glyphs for the trained models. We also illustrate the gradient backpropagation approach and discuss the interpretability of the learned representations by the CNN models. Finally, we ask the non-experts to rate the Grad-CAM visualizations of two models in terms of their level of interpretability. To the best of our knowledge, our study, specifically interpreting the CNN decisions for glyph classification and subjective assessment of the CNN visualizations, is novel in Digital Humanities and Archaeology context. 


\section{DATA}

To familiarize the reader with the studied data, this section first introduces the ancient Maya script, and then gives the details of the segmented Maya glyphs dataset used in the experiments.

\subsection{Maya Writing System}

The Maya writing system is quite visual, and is composed of complex logograms and syllabograms as opposed to stroke-based or continuous scripts of other languages [41]. In a recent catalog, Macri and Looper categorizes Maya glyphs into semantic groups such as animals, body parts, and faces [25]. Other categories are not straightforward to interpret as everyday objects, however they are also classified with some visual hints like square contour, with or without inner symmetry, elongated shapes, or variable number of components.

As with natural objects, samples from Maya glyph categories may exhibit high within-class variance and low between-class differences. Due to the historical period, place changes, and artistic reasons, Maya glyphs from one category may look relatively different to the exception of some specific "diagnostic" parts [17]. Similarly, between two similar-looking classes, the difference may be quite subtle, like changing diagnostic local parts such as eyes or teeth in human or animal head signs. Nevertheless, learning global patterns like shape contours (rectangular, head-shape, elongated) or local patterns (small circles, eyes, teeth) across classes, as CNN are truly capable of achieving, would benefit the recognition task.

\subsection{Crowdsourced Maya Glyph Segments}

In [3], a Maya Codical dataset was curated by crowdsourcing segmenting each glyph from glyph-blocks in ancient codices that survived until today (Dresden, Paris, Madrid). As these codices are from the post-classical era, within-class variance is relatively lower than the one for the monumental glyphs coming from different historical periods. However, it is possible to observe stylistic differences. The dataset is quite challenging as the number of samples per class is low, due to the lack of data.

Furthermore, the visual differences can be quite subtle. Fig. 1 illustrates samples from the ten classes with the highest number of samples per class. For example, the only visual difference between the first two and the last two glyphs in the top row is the orientation. These examples demonstrate that the classification task is not straightforward, even in the 10-class case with medium-scale data.

\section{METHODOLOGY}

To tackle the challenging glyph recognition task from the Maya codices, we used the deep CNNs that are able to learn powerful representations for various computer vision tasks. In the scope of our first and second research questions, we experimented with different training strategies and CNN architectures. In the scope of our third and fourth research questions, we worked on understanding and qualitatively assessing the representations that are learned by CNNs on glyph classification task. Thus, we followed two main steps in our methodology: (1) training a deep $\mathrm{CNN}$ to learn glyph representations, and (2) evaluating the visualizations of the discriminative glyph parts in a crowdsourcing task.

\subsection{Learning Glyph Representations (RQ1 \& RQ2)}

To learn discriminative glyph representations, we considered three approaches (i) assessing features learned by a pretrained network, (ii) transferring knowledge from an existing network through fine-tuning, (iii) full network training. Fig.2 illustrates our data-driven approaches. The first two cases are a good alternative to the third one when dealing with the challenge of small amount of data, as in our case.

4.1.1 Pretrained Features. Method. Given a pretrained network, and following [16], we use the output of the last convolutional or residual block from all glyph images in the training set as training features of a one-layer 


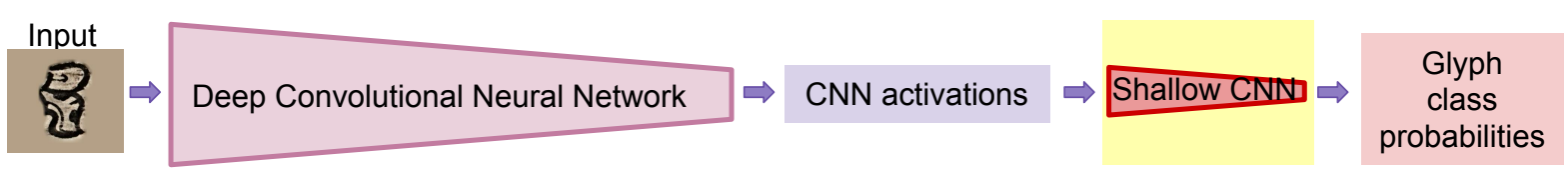

(a) Assessing pretrained CNN features.

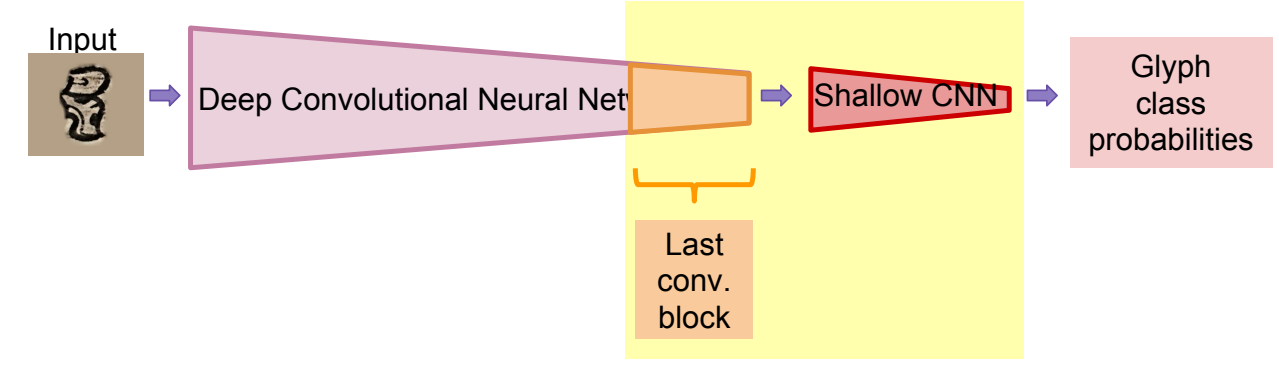

Fine-tune

(b) Fine-tuning the last convolutional block.

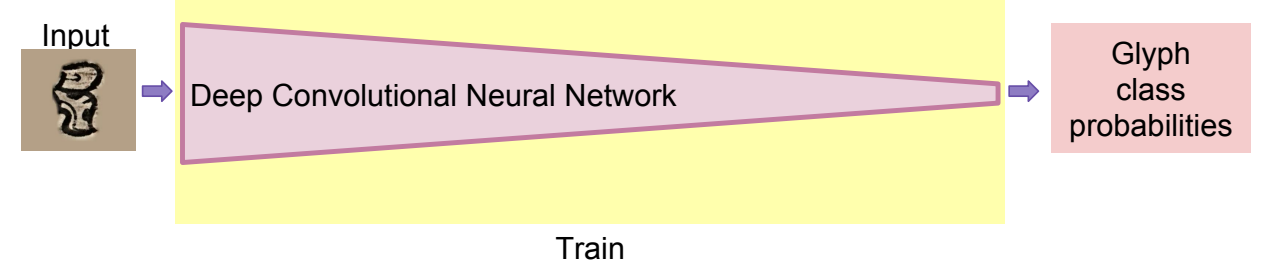

(c) Full network training.

Fig. 2. Three data-driven methods for supervised glyph classification. In each method, only the highlighted part of a CNN model was trained.

convolutional network (denoted as $B$ ). As depicted in Fig. 3a, this shallow network is composed of 1x1 convolution layer with 512 feature maps followed by batch normalization, ReLU nonlinearity, dropout, and a softmax classifier. Compared to the alternative approach of fine-tuning the fully-connected (FC) block of the pretrained net with our own data, this is much less costly.

Considered Networks. As pretrained networks, we chose the VGG-16 [37] and ResNet-50 (R50) [13] pretrained on ImageNet dataset, and the Sketch-a-Net (SaN) [43] pretrained on 250-class binary sketches [10].

As Simonyan et al. [37] showed that their deep CNN with 16 layers (VGG-16) performs similar to the one with 19 layers (VGG-19), we utilized VGG-16 in our experiments. We also experimented with the state-of-the-art residual network that uses BN layers and residual connections. On the other hand, the SaN is an altered version of the AlexNet for handling the sparse strokes with larger convolution kernels in the first layers. As there are fewer feature maps in the convolutional layers, this network has around $8.5 \mathrm{M}$ parameters compared to the $60 \mathrm{M}$ parameters of the original AlexNet.

For the VGG-16 and R50 models, we utilized existing pretrained models. However, due to the modifications we applied in the network, we trained the single-scale version of SaN from scratch. It is a 3-channel version of SaN for the populated colored versions of the sketch images (namely, sketch-RGB dataset) with three static background colors that are used to populate glyph images in [3]. One important difference of our re-trained 


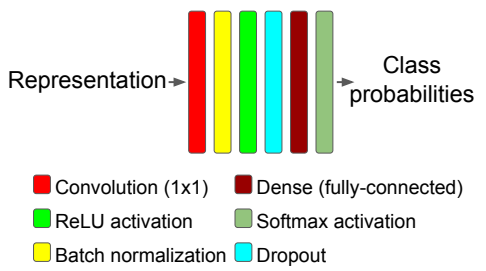

(a)

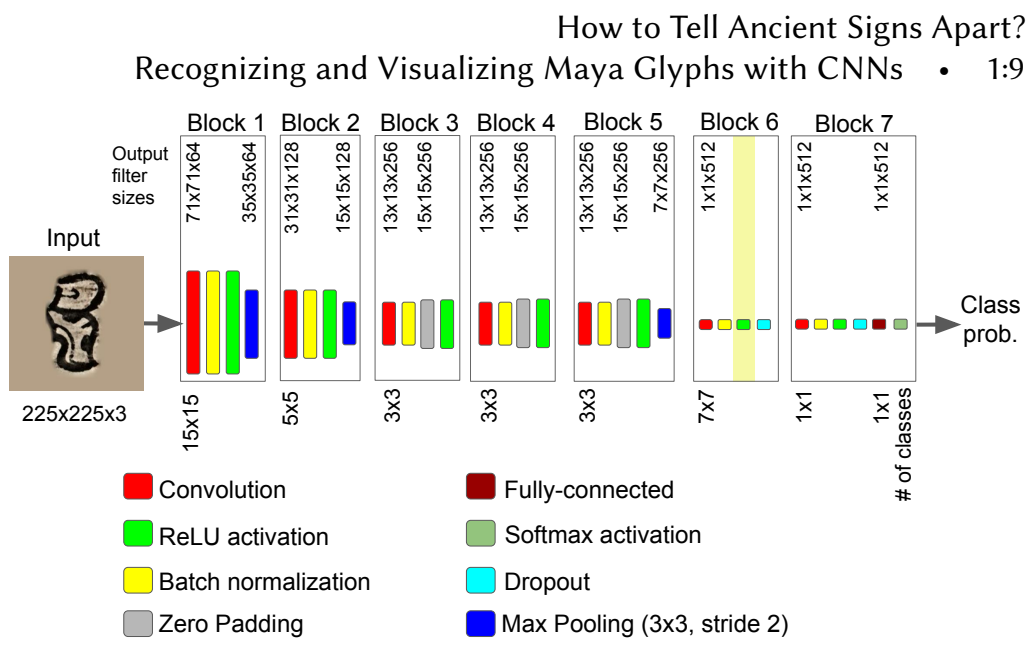

(b)

Fig. 3. (a) Shallow CNN model (B) that is used in Section 4.1.1, (b) Modified Sketch-a-Net model.

$\mathrm{SaN}$ is the $\mathrm{BN}$ [19] after each convolutional layer (see Fig. 3b). $\mathrm{BN}$ is shown to reduce covariate shift in the data during training, improve performance, and shorten the training time considerably. Furthermore, we utilized the Adam adaptive gradient update scheme [20] instead of stochastic gradient descent (SGD), which was shown by Kingma et al. [20] to outperform other adaptive SGD-based optimizers, as the gradients get sparser at the end of the optimization. With these configurations, we obtained $71.4 \%$ average test accuracy for 9750 colored sketches from the 250 classes (a random 1/6th split of the $60 \mathrm{~K}$ data, as another $1 / 6$ th is used for validation and the rest $2 / 3$ th is used for training).

The feature dimensions extracted from the pretrained nets are 2048 for the R50 (just before the fully-connected layer), $512 \times 7 \times 7=25088$ for the VGG-16 (output of the $5^{\text {th }}$ block), and 512 for the SaN (output of the $6^{\text {th }}$ block).

4.1.2 Network Adaptation. This method consists in jointly fine-tuning the last block of the pretrained net along with the weights of the shallow network B. More precisely, we replace the dense (fully-connected) layer at the end of the original network with the shallow network described in the Section 4.1.1. After freezing the weights in the original network up to the last block, we train the whole "stitched" model altogether with the glyph images. Specifically, the $6^{\text {th }}$ block in the Sketch-a-Net (SaN) pretrained model (see Fig. 3b), the $5^{\text {th }}$ convolutional block in the VGG-16, and the $5^{\text {th }}$ residual block in ResNet50 (R50) are fine-tuned.

For the shallow network at the top, we start from the weights trained as in Section 4.1.1, allowing the optimizer to start from a more relevant initialization than a random one. Additionally, the optimizer learning rate is set smaller than in Section 4.1.1, to prevent large gradient updates and disrupt the pretrained weights within the last convolutional block.

This fine-tuned network is denoted as $F$.

4.1.3 CNN training. We also investigated training of CNNs from scratch. Given our amount of data, networks with fewer parameters are preferable. As first choice, we utilized the classic LeNet model, however with ReLU activations, additional batch normalization after convolutional layers, and dropout strategy. This modified LeNet model has $44 M$ parameters to learn. Secondly, the sketch-specific SaN with additional BN layers was trained. Our third choice is the recent residual networks. Considering the number of parameters, we decided to train two versions of the ResNet with 18- and 50-layers (11M and $25 \mathrm{M}$ parameters respectively).

Another commonly-adopted option would be to train an inception network. Although the inception-v4 model has similar number of parameters, it has outperformed the R50 on Imagenet data for top-1 accuracy [38]. However, 
Canzani et al. [5] have shown that the contribution of number of parameters to top-1 accuracy is higher for the R18 and R50 models compared to the inception-v4 model. This implies that the information density stored in the neurons of these residual networks are higher than the inception-v4 model. This accuracy vs. parameter analysis is especially important for training a CNN from scratch with small- to medium-scale data. Therefore, we omit the inception-v4 model comparison here.

\subsection{Visualizing Discriminative Glyph Parts (RQ3 \& RQ4)}

4.2.1 Visualization with CNNs. To understand the learned CNN representations, we utilized both gradient backpropagation [36] and Grad-CAM [34]. With these methods, we visualized where the salient and the discriminative parts of the glyphs are for the model. Another use-case of such a class-activation visualization is to localize the glyphs in glyph-blocks (cluttered scenes) given the model trained on the desired glyph class.

Salient point visualization. After a single forward pass of the input through the network, partial derivatives of predicted class score w.r.t. pixel intensities are backpropagated and visualized [36]. This corresponds to visualizing the importance of input pixels such that the predicted class score gets influenced the most in case of a change in the input intensities.

CAM. Class Activation Mapping [45] is defined on a CNN that ends with a block of "convolutional layer $\rightarrow$ global average pooling layer $\rightarrow$ softmax layer". Hence, this visualization approach requires re-training of the weights after changing the $\mathrm{CNN}$ architecture, i.e. by replacing the layers after the last convolutional layer with Global Average Pooling (GAP) and a softmax layer. Then, the class score is obtained by a forward pass of the activations from the last convolutional layer:

$$
y^{c}=\sum_{k} w^{c} \frac{1}{Z} \sum_{i} \sum_{j} A_{i j}^{k}
$$

The localization map $L^{c} \in \mathbb{R}^{u \times v}$ for class $c$ with input width $u$ and height $v$ is computed as a linear combination of $k$ feature map activations $A^{k}$ and the re-trained weights $w_{k}^{c}$ between the Global Average Pooling layer and the softmax layer:

$$
L^{c}=\sum_{k} w_{k}^{c} A^{k}
$$

Grad-CAM. Being a generalization of CAM (except the final ReLU operation), GradCAM does not require a change in the CNN architecture and it is applicable to other neural network architectures as well [34].

Fig. 4 illustrates the GradCAM method. Essentially, first, the backpropagated gradients $\frac{\partial y^{c}}{\partial A_{i j}^{k}}$ are global-averagepooled and the importance weights $\alpha_{k}^{c}$ of feature map activations $A^{k}$ are obtained:

$$
\alpha_{k}^{c}=\frac{1}{Z} \sum_{i} \sum_{j} \frac{\partial y^{c}}{\partial A_{i j}^{k}}
$$

These weights correspond to the re-trained weights $w_{k}^{c}$ in CAM approach. Secondly, the linear combination of these weights and the feature map activations $A^{k}$ are computed. Finally, this linear combination is passed through a ReLU activation so that only positive activation-gradient combinations are considered.

$$
L^{c}=\operatorname{ReLU}\left(\sum_{k} \alpha_{k}^{c} A^{k}\right) .
$$


How to Tell Ancient Signs Apart?

Recognizing and Visualizing Maya Glyphs with CNNs • 1:11

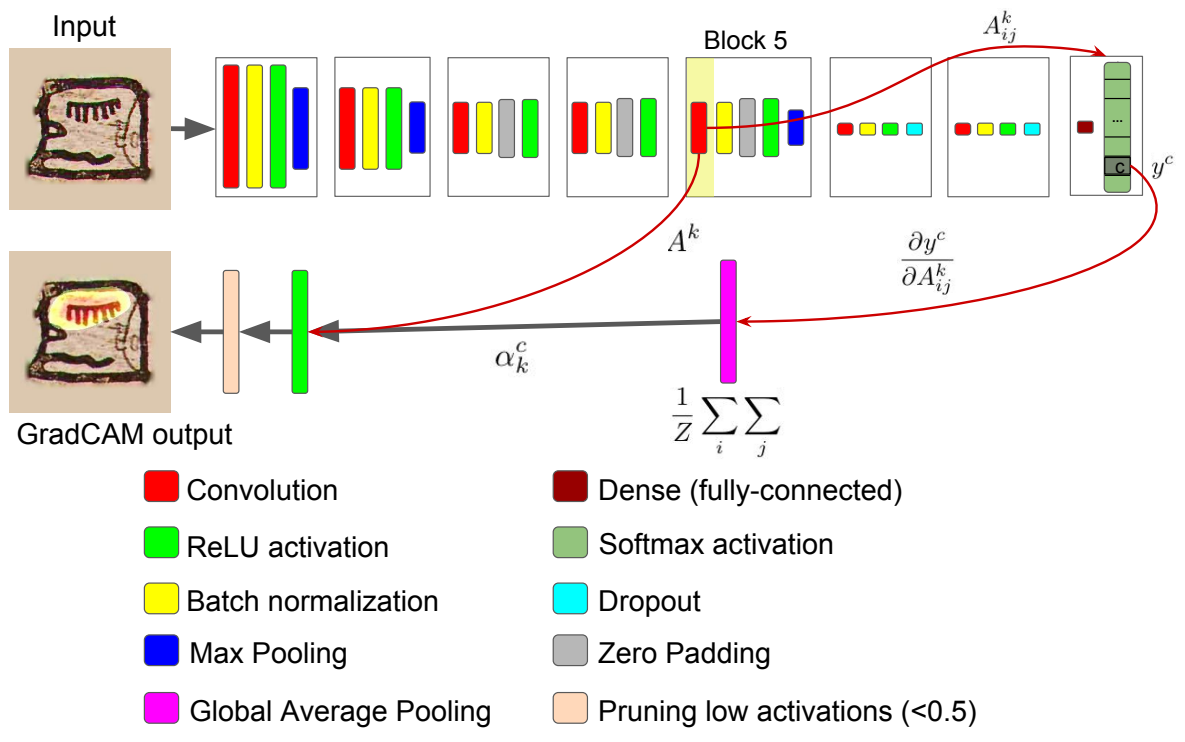

Fig. 4. Illustration of the GradCAM method.

In our case, to pay attention only to the most characteristic glyph parts, as a final operation, we eliminated the weak activation-gradient combinations (lower than 0.5 ) in the localization map.

4.2.2 Qualitative Crowdsourcing Analysis on CNN Visualizations. To assess the interpretability of the CNN representations qualitatively, we performed a preliminary crowdsourcing study. Specifically, this study is a perceptual comparative analysis of the visual representations of a sketch-specific netowrk (Sketch-a-Net) and a residual network (ResNet-50). These representations were trained on 50 classes of individual glyphs that were obtained via the crowdsourcing process described in our recent study [3].

As opposed to crowdsourcing studies with every-day objects in natural images, we have the challenge of non-experts not having a predefined concept of glyph categories. Thus, in our task design, we prepared detailed instructions and provided supervision to non-experts. Our focus is on whether non-experts' perception is at all aligned with the automatic discriminative models. We also address the research question of which CNN produces more appealing visual explanations according to the crowd (a sketch-driven network or a residual network).

Task Design. The main challenge of our crowdsourcing task is the non-familiarity of the data. Thus, in the task design, we provided supervision to non-experts in two ways. Considering that humans are good at generalizing from few samples, we provided a couple of examples that belong to the same class as the target glyph. As illustrated in the top parts of Fig. 6, in these examples, we also marked their possible diagnostic parts. Furthermore, as we hypothesize that people are good at relative analysis, we provided examples from other classes as well. In the task instructions, as shown in Fig. 5, we provided an example from the 50 classes used in training the CNN models. In the instructions, we also provided detailed explanation of the ratings and what they visually correspond to. The task itself is composed of two parts. In the first part, the annotator observes a visualization of the CNN output and rates it in a scale of 7 ranging from "very poor" to "very good". We ask the annotator to rate each of the networks' visualizations separately. In the second part, we show the visualizations from the two networks on the same glyph image, and ask the annotator to rate them relative to each other. 


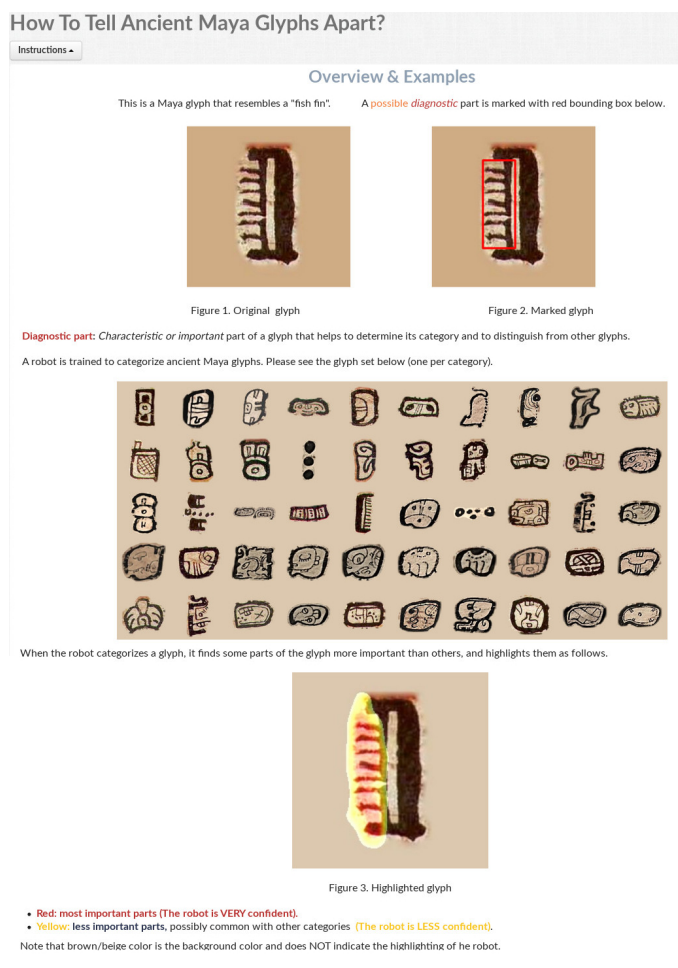

(a)

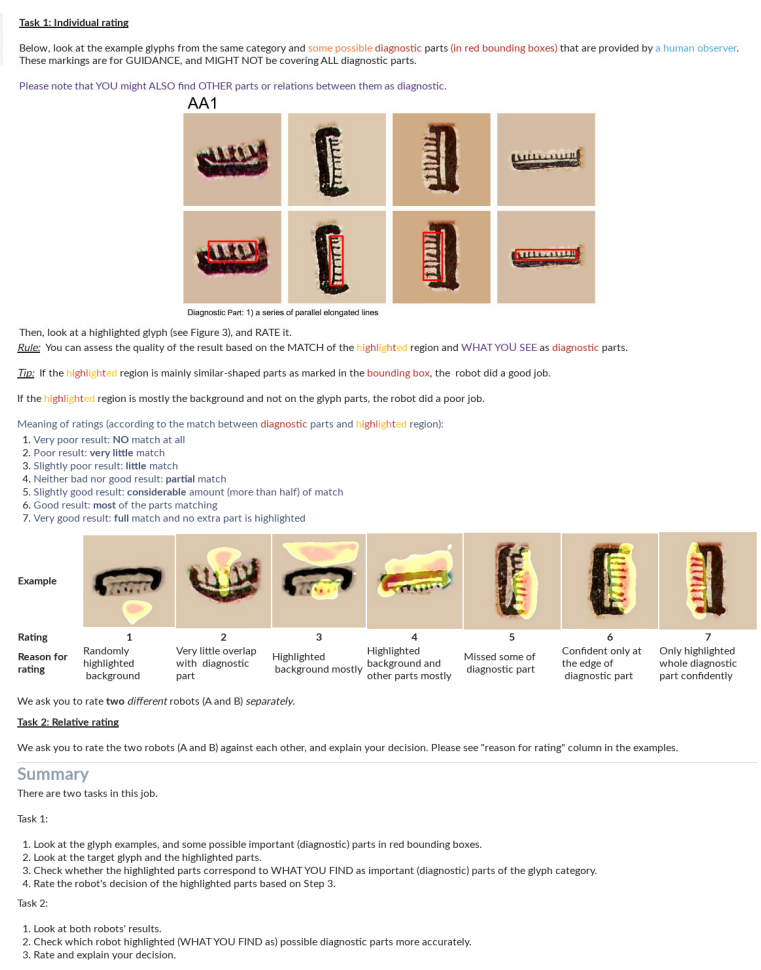

(b)

Fig. 5. Instructions of the crowdsourcing task: (a) introductory part, (b) task-specific part and the summary.

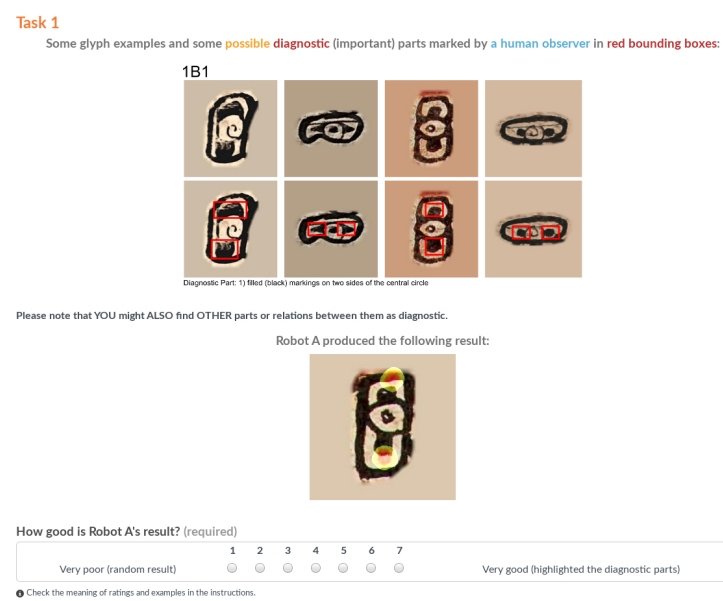

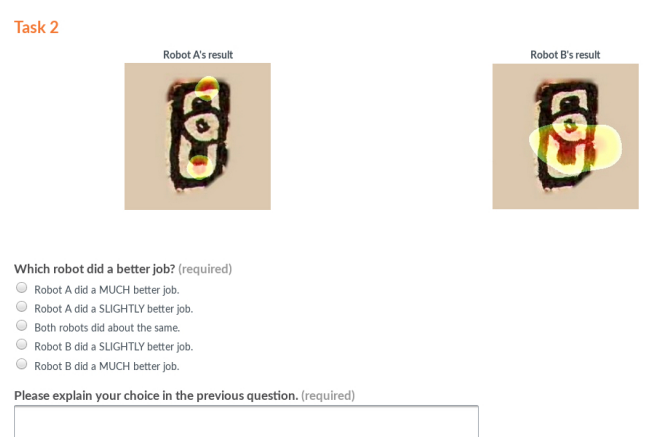

(b) Second task: Relative rating.

(a) First task: Individual rating.

Fig. 6. Two parts of the crowdsourcing task: (a) individual rating of a $\mathrm{CNN}$ visualization, (b) relative rating of two $\mathrm{CNN}$ visualizations against each other. 
How to Tell Ancient Signs Apart?

Recognizing and Visualizing Maya Glyphs with CNNs • 1:13

Table 2. Number of glyphs for the classification tasks.

\begin{tabular}{|c|c|c|c|c|c|}
\cline { 3 - 6 } \multicolumn{2}{c|}{} & \multicolumn{4}{c|}{ Number of classes } \\
\cline { 3 - 6 } \multicolumn{2}{c|}{} & $\mathbf{1 0}$ & $\mathbf{5 0}$ & $\mathbf{1 0 0}$ & $\mathbf{1 5 0}$ \\
\hline \multirow{3}{*}{$\begin{array}{c}\text { Number } \\
\text { of } \\
\text { samples }\end{array}$} & min & 211 & 50 & 20 & 5 \\
\cline { 2 - 6 } & mean & 255.7 & 132.66 & 81.19 & 57.74 \\
\cline { 2 - 6 } & median & 235 & 101 & 50 & 27 \\
\cline { 2 - 6 } & total & 2557 & 6633 & 8119 & 8661 \\
\hline
\end{tabular}

\section{EXPERIMENTAL SETTINGS AND RESULTS}

In this section, first, we provide the experimental settings and then discuss the results for the two main steps in our methodology (glyph classification with CNNs in the scope of RQ1 \& RQ2 and qualitative assessment of the visual CNN representations in a crowdsourcing task in the scope of RQ3 \& RQ4).

\subsection{Experimental Settings for Classification (RQ1 \& RQ2)}

Data. We use the dataset introduced in [3] and shared by the authors, which consists of Maya glyph segments curated via crowdsourcing. The authors point out that data imbalance is one of the challenges of the dataset. Accordingly, they prepared several classification cases (easy to difficult) with different number of classes and number of samples per class. Table 2 presents the available minimum, mean, and maximum number of segments for these cases. Note that these glyph segments are populated with four background colors $\left(3\right.$ static $^{1}, 1$ dynamic RGB color) afterwards. In this paper, we are using all available samples in the selected 150 classes instead. Different from the original paper, where the authors undersample the data for creating a balanced set [3], we oversample the existing examples such that each class has 1000 training, 300 validation, and 300 testing samples. Therefore these oversampled sets are a mix of original data and synthetic data, as the newly-added synthetic samples are obtained by applying random geometric transformations as detailed below.

Data preparation and augmentation. For all the experiments, we use the oversampled data created with random geometric data augmentation, comprising rotation (within $[-15,15]$ degrees), vertical and horizontal translation $(+/-0.1 \times$ image width), and zooming (scale within $[0.8,1.2])$. We applied only these traditional transformations in order not to alter the the nature of the data. In method 1, we only rely on this already-augmented and oversampled examples. However, during fine-tuning (method 2) and training the CNNs from scratch, we re-apply this augmentation on-the-fly on the already-oversampled data, while constructing the batches with random sampling. Similar to the original papers, the image width is set to 224 pixels when using the VGG-16, ResNet, and LeNet models; whereas, for the SaN case, it is set to 225 pixels.

Tasks and performance measures. We focus on four tasks: 10-, 50-, 100-, and 150-class glyph classification. Note that the 10,50, and 100 classes are chosen from the 150-class set such that they have the most number of samples per class. We report the average test accuracy (top-1) along with the top-5 accuracy (i.e. we consider the prediction as true positive if the true class is among the top-5 predicted classes). Test accuracy is defined as follows: $\frac{t p+t n}{N_{\text {dataset }}}$, where $t p$ denotes the number of true positives, $t n$ denotes the number of true negatives, and $N_{\text {dataset }}$ is the number of all samples in the dataset.

Training. For training the shallow net over pretrained features and training the CNNs from scratch, Adam optimizer is used with the learning rate $10^{-5}$ and $10^{-4}$ respectively. For fine-tuning, Stochastic Gradient Descent (SGD) with momentum (0.9) is used. The learning rate is set to $10^{-4}$, and is reduced with a factor of 0.2 in case the validation loss is not decreasing for 10 epochs. We applied model check-pointing (keeping track of the parameters that result in highest validation accuracy during the optimization) during all the training cases. For pretrained

\footnotetext{
${ }^{1}$ Chosen RGB colors are $(180,160,135)$ for Dresden Codex, $(220,200,175)$ for Madrid Codex, and $(210,185,160)$ for Paris Codex.
} 
net training and fine-tuning, the maximum number of epochs is set to 500 empirically, whereas for training from scratch, we followed an early-stopping approach with a patience factor of 20 epochs, i.e. terminating training if validation loss does not decrease for 20 epochs.

\subsection{Experimental Settings for Crowdsourcing (RQ3 \& RQ4)}

Data. In this preliminary study, we limited our dataset to 10 glyphs from 10 classes. We computed the Grad-CAM results of two networks for these randomly-chosen 100 glyphs. To make the visualizations more selective, we eliminated the weak activations in these mappings (empirical threshold is set as 0.5 in the range of $[0,1]$ ).

In an attempt to understand the visualizations on this set of chosen glyphs, as a preliminary check, we performed a pixelwise comparison between these visualizations and the manually-marked groundtruth masks. We observed that the pruned ResNet-50 visualizations overlap with the groundtruth masks in all cases, whereas the Sketch-a-Net visualizations overlap with the groundtruth masks for 98 out of the 100 glyphs. Furthermore, the ResNet-50 visualizations were more diffused with higher average recall (0.754 vs. 0.582$)$ and lower average precision values ( 0.255 vs. 0.431$)$.

Over this set of chosen glyphs, to make the perceptual task easier for non-expert observers, we used doublecolored visualizations. In these visualization, yellow color can be considered to correspond to "important" and red color to "very important" parts. Below, we give the details of our crowdsourcing task design.

Task Settings. Using the Crowdflower terminology introduced in [3], we set 4 tasks in a page, and paid an annotator 10 USD cents per page. We collected annotations from 10 annotators per task. We set 10 test questions to be used in quiz mode. Quiz mode enables to eliminate spammers or low-performing annotators. In total, we collected 1000 annotations (10 for each of the 100 glyphs).

\subsection{Classification Results (RQ1 \& RQ2)}

We experimented with three main training strategies, and with a range of different models as follows: 1) training a shallow neural network with the pretrained features, 2) fine-tuning the last block of Sketch-a-Net with batch normalization pretrained on RGB populated sketch images (SaN-B or SAN-F); VGG-16 net pretrained on ImageNet (VGG-B or VGG-F); ResNet-50 pretrained on ImageNet (R50-B or R50-F); or 3) training from scratch LeNet with batch normalization (L-S); Sketch-a-Net with batch normalization (SaN-S); ResNet-18 (R18-S); and ResNet-50 (R50-S).

Table 3 presents the results obtained with these methods. Except for the 10-class case of the SaN models, we observe 2.0 to $28.6 \%$ absolute improvement in top-1 average accuracies of the fine-tuned $F$ models compared to the corresponding pretrained $B$ models. Especially, R50 benefits highly from fine-tuning. The most notable point in Table 3 is the outperforming performances of the SaN and R50 models trained from scratch. These models perform consistently better than their corresponding fine-tuned models.

For training from scratch, with classic sequential networks, the SaN model that is deeper and has fewer parameters than LeNet, always performs better (10.4 to 24.3\%). However, performances of the residual nets are similar, and the marginal differences do not allow us to conclude that the shallower net with fewer parameters always perform better with this special network design (as the identity mapping residual connections help the network behave as if it had dynamic depth).

Table 4 emphasizes our contribution in this paper that we achieved with oversampled training sets. For brevity, we only presented the results from Sketch-a-Nets that were trained from scratch with different configurations. Compared to the configurations in [4] that used original training set, we achieved a significant improvement in average class accuracies on the original test sets (last row of Table 4). Thanks to using oversampled balanced training sets, the batch generation during training was fair to all the classes, and even the classes with few samples contributed to the optimization process equally. This resulted in much higher average class-based accuracies. 
How to Tell Ancient Signs Apart?

Recognizing and Visualizing Maya Glyphs with CNNs • 1:15

Table 3. Average test top-1 (T-1) and top-5 (T-5) accuracies. Models: L: LeNet, SaN: Sketch-a-Net, R: ResNet, VGG-16. Conditions: B: pre-trained, F: fine-tunning, S: learned from scratch. Best performances in B, F, and S are in bold.

\begin{tabular}{|c|c|c|c|c|c|c|c|c|}
\cline { 2 - 10 } \multicolumn{1}{c|}{} & \multicolumn{8}{c|}{ Number of classes } \\
\cline { 2 - 10 } \multicolumn{1}{c|}{} & \multicolumn{2}{|c|}{$\mathbf{1 0}$} & \multicolumn{2}{c|}{$\mathbf{5 0}$} & \multicolumn{2}{c|}{$\mathbf{1 0 0}$} & \multicolumn{2}{|c|}{$\mathbf{1 5 0}$} \\
\hline Model & T-1 & T-5 & T-1 & T-5 & T-1 & T-5 & T-1 & T-5 \\
\hline SaN-B & 81.5 & 98.0 & 65.2 & 85.8 & 50.5 & 71.2 & 42.3 & 62.7 \\
\hline VGG-B & $\mathbf{8 7 . 9}$ & $\mathbf{9 9 . 1}$ & $\mathbf{7 7 . 1}$ & $\mathbf{9 1 . 9}$ & $\mathbf{6 2 . 4}$ & $\mathbf{8 1 . 3}$ & $\mathbf{5 2 . 8}$ & $\mathbf{7 4 . 0}$ \\
\hline R50-B & 77.8 & 96.4 & 54.3 & 79.0 & 50.3 & 75.0 & 31.8 & 52.8 \\
\hline \hline SaN-F & 81.0 & 98.5 & 70.9 & 90.1 & 58.7 & 80.3 & 50.2 & 72.7 \\
\hline VGG-F & $\mathbf{8 9 . 9}$ & 98.9 & $\mathbf{8 5 . 4}$ & $\mathbf{9 5 . 9}$ & $\mathbf{7 3 . 5}$ & $\mathbf{8 9 . 2}$ & $\mathbf{6 4 . 5}$ & $\mathbf{8 3 . 5}$ \\
\hline R50-F & 87.0 & $\mathbf{9 9 . 3}$ & 79.4 & 93.9 & 70.5 & 87.7 & 60.4 & 78.9 \\
\hline \hline L-S & 81.4 & 98.2 & 70.1 & 89.4 & 52.2 & 74.9 & 46.0 & 68.0 \\
\hline SaN-S & $\mathbf{9 1 . 0}$ & $\mathbf{9 9 . 8}$ & $\mathbf{8 7 . 8}$ & 95.9 & 75.1 & 90.1 & $\mathbf{7 0 . 3}$ & 85.4 \\
\hline R18-S & 88.0 & 99.6 & 87.3 & $\mathbf{9 6 . 9}$ & 78.6 & 94.3 & 68.6 & $\mathbf{8 5 . 5}$ \\
\hline R50-S & 89.1 & 99.6 & 85.1 & 96.6 & $\mathbf{7 9 . 2}$ & $\mathbf{9 5 . 3}$ & 67.4 & 84.6 \\
\hline
\end{tabular}

Table 4. Average sample-based top-1 (T-1) accuracies, and average class-based accuracies (CA) for the original test set.

\begin{tabular}{|l|c|c|c|c|c|c|c|c|}
\multicolumn{1}{c|}{} & \multicolumn{9}{c|}{ Number of classes } \\
\cline { 2 - 8 } \multicolumn{1}{c|}{} & \multicolumn{2}{|c|}{$\mathbf{1 0}$} & \multicolumn{2}{c|}{$\mathbf{5 0}$} & \multicolumn{2}{c|}{$\mathbf{1 0 0}$} & \multicolumn{1}{c|}{$\mathbf{1 5 0}$} \\
\hline \multicolumn{1}{c|}{ Method } & T-1 & CA & T-1 & CA & T-1 & CA & T-1 & CA \\
\hline SaN-S trained with original set $[4]$ & 91.7 & 77.1 & 89.1 & 51.9 & 86.7 & 26.9 & 84.2 & 16.0 \\
\hline SaN-S trained with original set and class weights $[4]$ & 90.7 & 75.7 & 89.7 & 52.4 & 87.2 & 26.8 & 84.8 & 17.7 \\
\hline SaN-S trained with oversampled set (this paper) & 91.1 & $\mathbf{9 1 . 1}$ & 89.0 & $\mathbf{8 8 . 9}$ & 87.0 & $\mathbf{8 3 . 8}$ & 84.4 & $\mathbf{7 3 . 6}$ \\
\hline
\end{tabular}

With this motivation, as hinted at the end of the discussion in [4], we decided to conduct all the experiments with oversampled balanced sets as reported in Table 3.

Fig. 7 shows the individual class accuracies (average test top-1) compared to the ratio of the original training samples for 150-class Sketch-a-Net trained from scratch. The blue line in the plot indicates the ratio of the number of original training samples in a class to the number of oversampled training samples in a class. (This ratio was presented as a percentage to be in the same scale with the accuracy -red line-.)

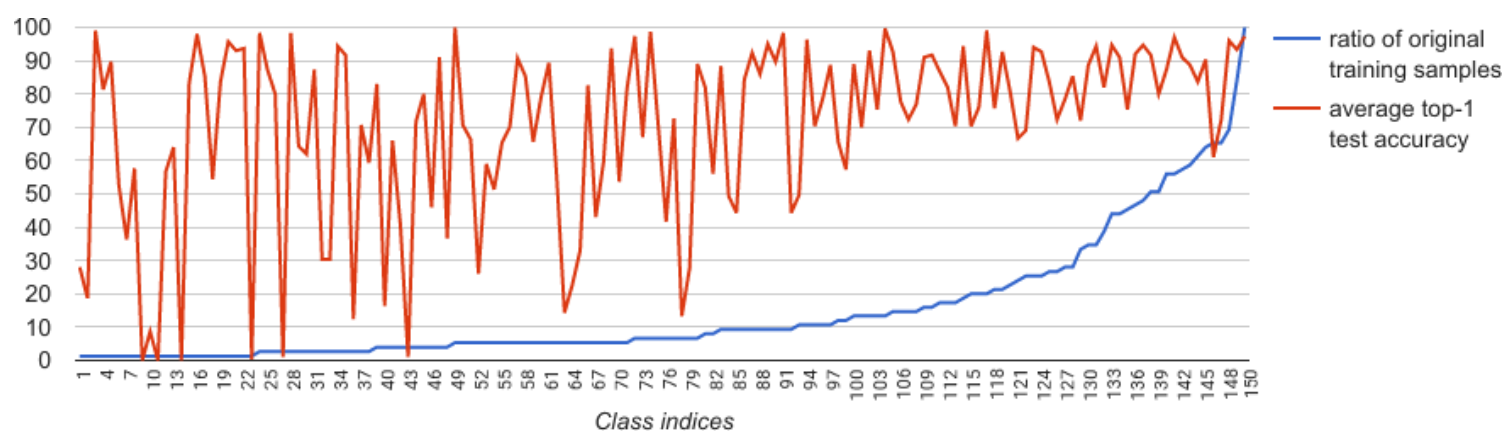

Fig. 7. The ratio of original training samples vs. average top-1 class accuracies for the SaN-S model for the 150 classes. Despite the performance fluctuations among classes, top 50 most frequent glyph classes exhibit a high performance trend, showing the importance of original training data size. 
Table 5. Visual examples of the classes whose performances got affected the most when the 50-class SaN-S or 150-class $\mathrm{SaN}-\mathrm{S}$ models were used.

\begin{tabular}{|c|c|c|c|c|}
\hline Class & $\begin{array}{l}\text { T-1 acc. with } \\
\text { SaN-S trained } \\
\text { on } 50 \text { classes }\end{array}$ & $\begin{array}{l}\text { T-1 acc. with } \\
\text { SaN-S trained } \\
\text { on } 150 \text { classes }\end{array}$ & \multicolumn{2}{|c|}{$\begin{array}{c}\text { Similar } \\
\text { classes in } \\
150 \text { class-set }\end{array}$} \\
\hline$\because$ & 95.3 & 78.3 & 8 & : \\
\hline 003 & & & 004 & 008 \\
\hline & 82.7 & 72.3 & & \\
\hline XE1 & & & XE3 & XE7 \\
\hline ㄷiiㅣ & 31.3 & 70.0 & 6 & \\
\hline $1 \mathrm{SD}$ & & & 1SC & \\
\hline
\end{tabular}

This plot demonstrates a lot of fluctuations in the class accuracies. We hypothesize this is due to the nature of the data, as some classes have small within-class variation and large between-class variation, so that they can be classified well even with small amount of training samples. The maximum number of training samples is 900 . Despite the fluctuations, we can observe the trend of increasing accuracy, especially when the number of original training samples is more than 96 (i.e. at least $10.67 \%$ of the populated 900 samples are original samples). This trend is more visible from the class index 94 and on.

Another question is how the performance on the existing classes get affected when a model is trained with additional classes and in general more data. We inspected the class accuracies of the 50 classes from the 50-class and 150-class SaN-S models. We observed that the performance for 9 classes dropped $10 \%$ or more, however the performance for 14 classes improved when trained within 150 classes. As expected, in the 150-class set, the classes in the 50-class set get more competitors that share local features. In the discussion that follows, we refer to signs using the Macri-Looper catalog naming system [25]. For instance, as illustrated in Table 5, due to the inclusion of other numerical signs, the performance of class 003 (literally 3 as three horizontal thick dots) drops. Similarly, sign XE1's performance drops due to more similar-looking classes coming to play such as signs XE3 and XE7 (all have dots and vertical parallel lines inside a square thick contour). Another notable difference is the $38 \%$ increase in the performance of sign 1SD whereas its competitor sign 1SC's performance drops by $17 \%$. This might be due to the inclusion of more classes, which forces the network to spot more subtle differences, or due to the inclusion of more head-signs (increasing in number from 4 to 29) so that the network becomes better at spotting "eyes" and distinguishing them from any other random circle (sign 1SD looks like a profile head sign with a prominent eye and mouth, whereas sign 1SC has two components: a head-like circle with three small inner circles, and a body with inner details similar to 1SD).

Summary of trends. From our experiments, we conclude that 1) VGG-16 seems to have more robust pretrained representations than the ResNet-50 features; 2 ) fine-tuning improves the results compared to the pretrained feature classification baseline; 3 ) oversampling is essential for handling imbalanced small-scale glyph datasets; 4) batch normalization and dropout enables training a $\mathrm{CNN}$ from scratch with medium-scale oversampled data, and outperforms fine-tuning results with a sketch-specific net. 
How to Tell Ancient Signs Apart?

Recognizing and Visualizing Maya Glyphs with CNNs • 1:17

Table 6. Diagnostic features of five Maya signs, commented by epigrapher experts. We present the discriminative parts inferred by the 50-class SaN-S model. The heatmaps are visualized via the guided backpropagation approach and the Grad-CAM method (red is for high, blue is for low response). Column 6 and 7 present the examples of weak localization of corresponding glyphs in the glyph-blocks. (+) indicates a correct correspondence (examples in column 6), and (-) indicates a wrong, uninformative, or partial-correspondence (column 7).

\begin{tabular}{|c|c|c|c|c|c|c|c|}
\hline $\begin{array}{c}\mathrm{T} \\
\text { class }\end{array}$ & $\begin{array}{l}\text { MV } \\
\text { class }\end{array}$ & $\begin{array}{l}\text { Diagnostic } \\
\text { Feature }\end{array}$ & $\begin{array}{l}\text { Salient } \\
\text { Points }\end{array}$ & Grad-CAM & $\begin{array}{c}\text { Grad-CAM } \\
\text { in a block }(+)\end{array}$ & $\begin{array}{c}\text { Grad-CAM } \\
\text { in a block (-) }\end{array}$ & $\begin{array}{c}\text { Class } \\
\text { Acc. }(\%)\end{array}$ \\
\hline$\sqrt{6}$ & $\stackrel{\mathbb{L e}}{\mathrm{HE} 6}$ & $\begin{array}{l}\text { An outer C-shaped frame, } \\
\text { two circles, } \\
\text { the "teeth" attached to } \\
\text { inner line of the frame. }\end{array}$ & & & $=$ & & 96.7 \\
\hline 017 & ZUJ & $\begin{array}{l}\text { A hook inside the central circle, } \\
\text { two to four vertical parallel lines } \\
\text { coming up from the central circle. }\end{array}$ & & & & & 96.0 \\
\hline$\left[\begin{array}{l}{[\mathrm{g}} \\
023\end{array}\right.$ & $\overbrace{1 G 1}^{80}$ & $\begin{array}{l}\text { Two notches/discontinuity } \\
\text { at the bottom of its outer shape, } \\
\text { inner thin line whose } \\
\text { two ends face downwards, } \\
\text { the "teeth" elements attached to } \\
\text { the inner line. }\end{array}$ & $\rightarrow$ & & & & 85.0 \\
\hline (लIII) & AA1 & $\begin{array}{l}\text { Parallel elongated lines, } \\
\text { framed by a thicker general outline. }\end{array}$ & & & & & 96.3 \\
\hline (2) & 8 & $\begin{array}{l}\text { General outline resembling a necklace } \\
\text { that is composed of } \\
\text { a precious stone in the middle, } \\
\text { and twines on both sides of it. }\end{array}$ & (28) & (6) & $\frac{0}{c 07}$ & $\begin{array}{l}509 \\
2501\end{array}$ & 88.3 \\
\hline
\end{tabular}

\subsection{Visualization and Interpretation Results (RQ3 \& RQ4)}

Table 6 illustrates samples from the Thompson (T) and Macri-Vail (MV) catalogs, as well as available expert comments about the diagnostic features of five glyph categories. We demonstrate the salient points obtained via [36], and the Grad-CAM heatmaps that correspond to the discriminative part of the glyph sample according to the trained model (i.e. the SaN trained from scratch with 50-classes); red means high and blue means low response. We also present the class accuracies of these specific classes obtained with this model. Besides, we present Grad-CAM responses of these glyph categories in the glyph-blocks for both positive (+); and uninformative, negative, or partial-correspondence (-) cases.

Table 6 shows that Grad-CAM is successful at capturing discriminative features for the shown glyphs, and it is also able to localize them in glyph-blocks, but fails in several cases as the negative examples show. As reported by others, gradient backpropagation is less informative than Grad-CAM.

Fig. 8 depicts examples of discriminative part heatmaps obtained via Grad-CAM according to the predicted class for the HE6 class (first glyph in Table 6). The top row illustrates successful visualizations that match the 


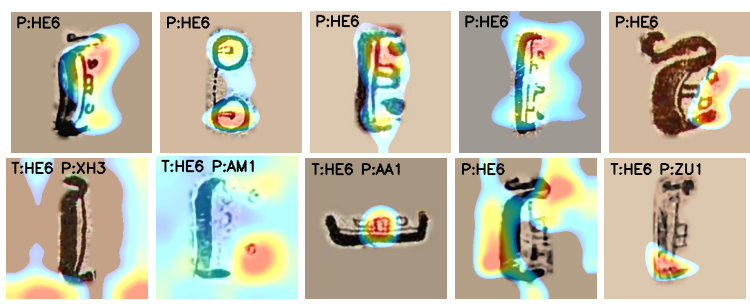

Fig. 8. Success (top row) and failure examples (bottom row) of activations of discriminative parts for sign HE6 with the 50-class SaN-S model. Positive activation from the model for each input image is illustrated as a colored intensity map (referred as heatmap) over the input image. In this map, blue indicates a weak, yellow indicates a medium-level, and red indicates a strong positive activation. The heatmaps are visualized via Grad-CAM based on the predicted class ("P") by the model for the given input image. If the model made a false prediction, the true class of the glyph image is also indicated (as "T"). The class labels are given in Macri-Vail catalog codes.

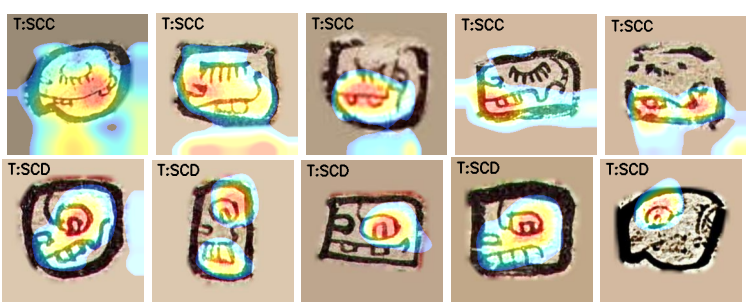

Fig. 9. The SCC (top row) and SCD examples (bottom row) and the activations of discriminative parts for the 50-class SaN-S model. The heatmaps are visualized via Grad-CAM method based on the true class ("T").

expert comments, whereas the bottom row shows failures of Grad-CAM. Note that all the top row examples are classified correctly, even the second example which is a different variant of the glyph and is not dominant in the training set. On the other hand, in the bottom row, we observe that the absence or different disposition of the two diagnostic dots end up in misclassification or not-so-well-localized heatmaps.

In the recent Macri-Vail Maya sign catalog [26], the SCC sign that means "death or dead" is described as "head with closed eye", whereas the SCD sign that corresponds to "death god" is described as "skull" or "skull with eyeballs". Fig. 9 depicts heatmaps of the true class activations of these glyphs (top row for SCC, bottom row for SCD). Notably, for the SCD examples, we observe that the attention of the CNN representation is on the "eyeball", yet for the SCC examples the nose and the teeth around the mouth are also highlighted. This is most likely due to the existence of other categories that showcase the "closed eye with eyelashes" as the main specificity.

Overall, we see that the use of Grad-CAM is promising to visualize diagnostic features of glyphs. How to systematize the evaluation of the visual explanations produced by deep networks is investigated in a preliminary crowdsourcing study. We describe the analysis of the crowdsourcing results in the next subsection.

Analysis of Crowdsourced Ratings. To understand the preference of crowdworkers among the visualizations of the studied two CNNs, we, first, analyzed the distributions of individual ratings from Task 1 (shown in Fig. 10a) with the Kolmogorov-Smirnov (KS) 2-sample test [27]. KS 2-sample test rejects the null hypothesis that samples come from the same distribution based on comparing the p-value with the significance level $\alpha$ [27]. In our case, KS test rejected the null hypothesis at significance level $\alpha=0.005$. This result suggested that the crowdworkers did not perceive the heatmaps from the two CNN models as the same.

Secondly, we analyzed the aggregated individual ratings of the glyph instances. To obtain these aggregated ratings, we simply averaged all the individual ratings from 10 annotators that were assigned to the annotation of that glyph. Complementary to the point above, in Fig. 10b, we observed that the percentage of the glyphs that is 
How to Tell Ancient Signs Apart?

Recognizing and Visualizing Maya Glyphs with CNNs • 1:19

Table 7. Aggregated results of interpretability crowdsourcing analysis on the pilot set for the first 5 classes. Blue frames indicate the crowdworkers' preference in relative ratings.

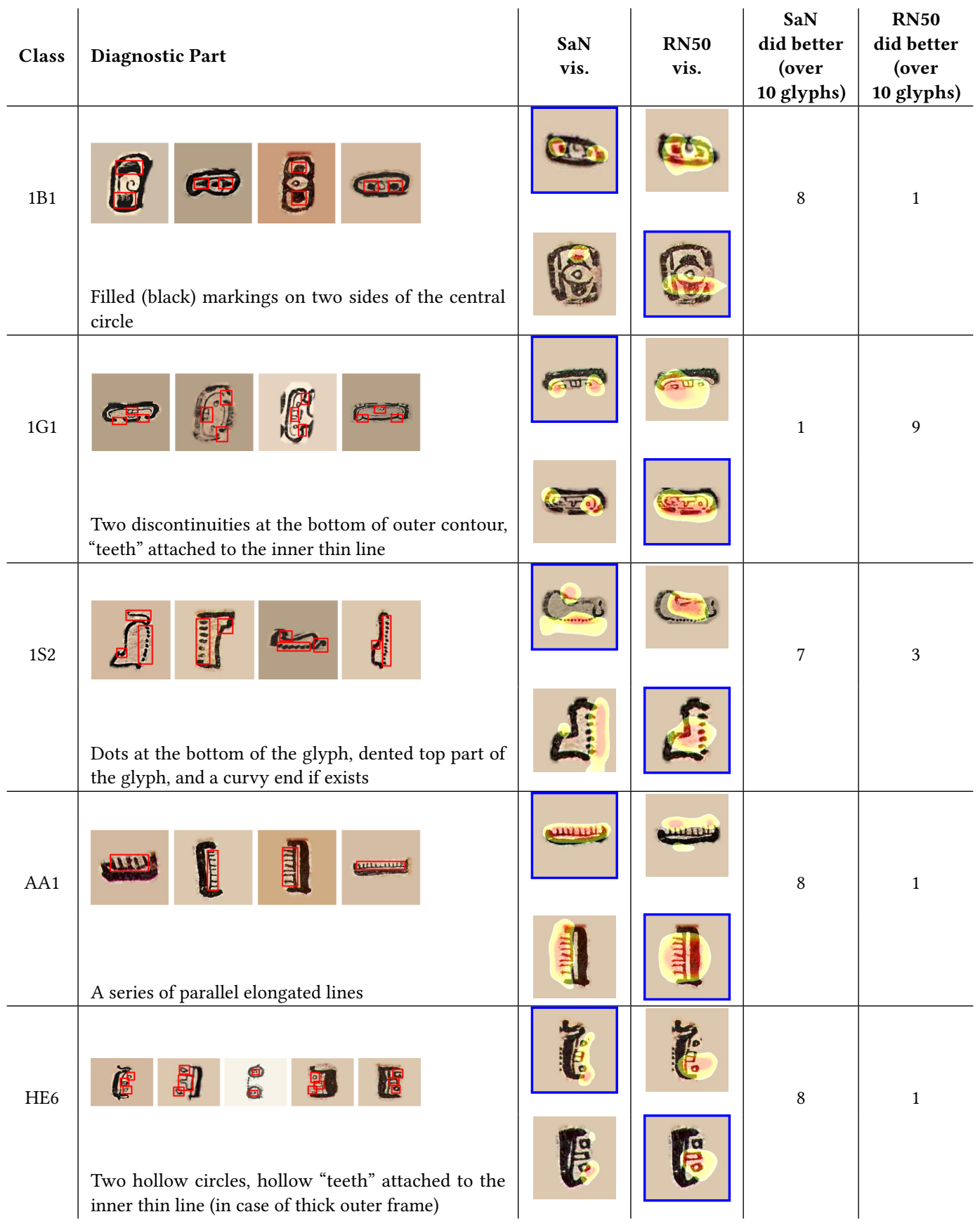


Table 8. Aggregated results of interpretability crowdsourcing analysis on the pilot set (continued for the rest of the 10 classes). Blue frames indicate the crowdworkers' preference in relative ratings.

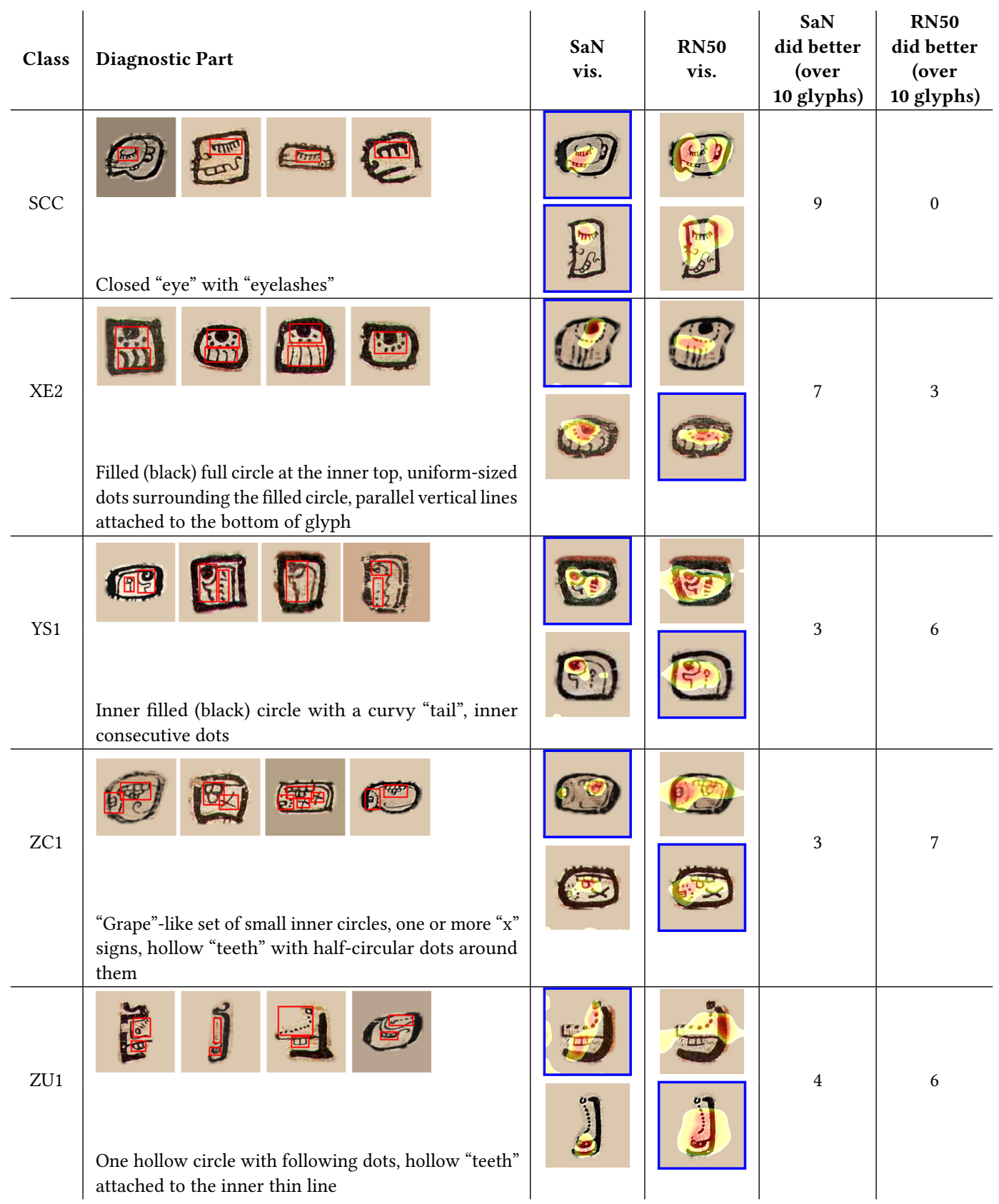


How to Tell Ancient Signs Apart?

Recognizing and Visualizing Maya Glyphs with CNNs 1:21

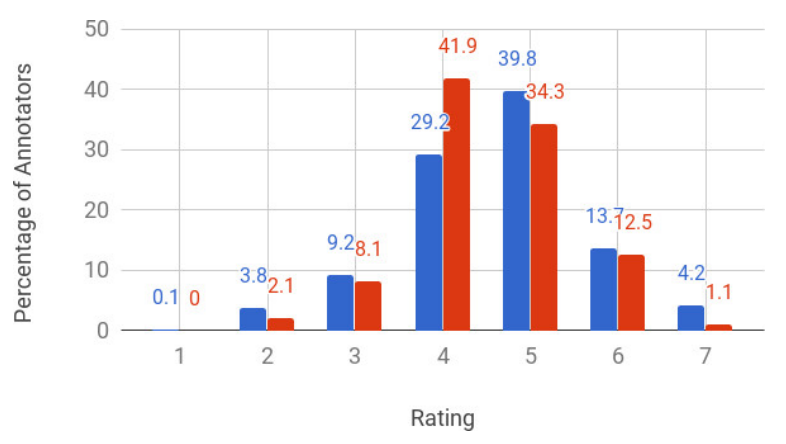

(a) Task 1. Individual ratings.

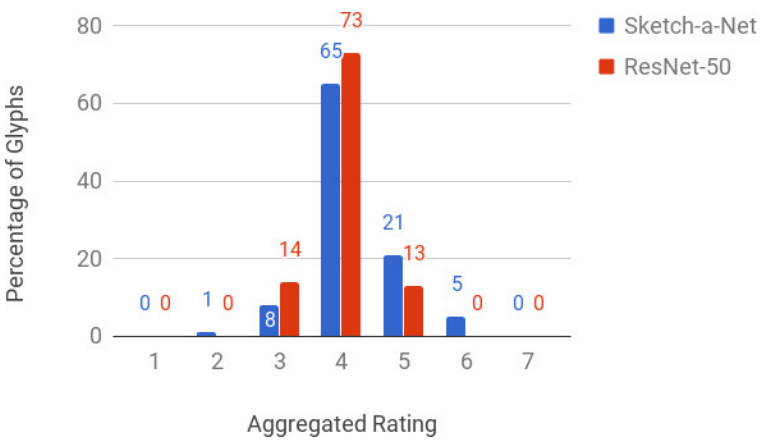

(b) Task 1. Aggregated individual ratings.

Which model did a better visualization?

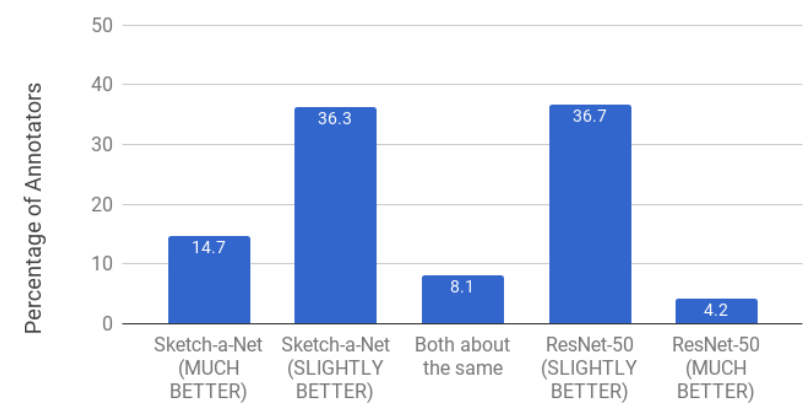

(c) Task 2. Relative ratings.

Fig. 10. The distributions of (a) individual ratings, (b) individual ratings aggregated per glyph, and (c) relative ratings in the pilot crowdsourcing study on interpretability of $\mathrm{CNN}$ representations.

rated as positive (rating 5, 6 or 7) in the overall aggregated ratings are higher for the Sketch-a-Net visualizations $(21+5=26 \%)$ than for the Residual Network visualizations $(13 \%)$.

Thirdly, to check the relative ratings from Task 2, we aggregated the relative ratings of 10 annotators per glyph in a weighted manner. The "much better" options (extreme end of the scale) get 2 votes whereas "slightly better" options get 1 vote. Overall, among the 100-glyphs, the crowdworkers favored the Sketch-a-Net visualizations over the ResNet-50 visualizations for 52 glyphs. ResNet-50 visualizations were favored for 41 glyphs. There was a tie for 7 glyphs. The last two columns of Table 7-8 show these distributions across classes. From these class-based results, we observed that the ResNet-50 visualizations were found more appealing for the categories with several diagnostic parts (i.e. ZC1, YS1, ZU1, and 1G1). In these categories, the diagnostic parts cover almost the whole glyph. Since the ResNet-50 heatmaps, in general, highlight more regions than the Sketch-a-Net heatmaps, we considered this finding plausible.

Finally, in Table 9, we listed a few of the insightful comments from the crowdworkers. These comments help to understand what kind of rating criteria were used by the crowdworkers.

\section{CONCLUSION}

Our paper addressed four research questions. For RQ1, we studied three training approaches with CNNs for a challenging Maya codical glyph dataset. To address RQ2, we assessed representations learned in several existing 
Table 9. Some of the comments from the crowdworkers on the interpretability of the two CNN visualizations (Sketch-a-Net -SaN- vs. ResNet50 - RN-) about the glyph diagnostic parts. In the comments, Robot A and B refer to the SaN and RN models, respectively. In the relative ratings, 0,1 , or 2 indicates "about the same," "slightly better," "much better," respectively.

\begin{tabular}{|c|c|c|c|c|}
\hline Class & Crowdworker Comment & $\begin{array}{c}\text { Ind. } \\
\text { rating } \\
(\mathrm{SaN})\end{array}$ & $\begin{array}{l}\text { Ind. } \\
\text { rating } \\
(\mathrm{RN})\end{array}$ & $\begin{array}{l}\text { Rel. } \\
\text { rating }\end{array}$ \\
\hline $\begin{array}{l}\text { 1B1 (top in } \\
\text { Table 7) }\end{array}$ & $\begin{array}{l}\text { Robot A was more specific in highlighting the most significant parts of } \\
\text { the glyph, while robot B highlighted most of the glyph }\end{array}$ & 6 & 4 & SaN_2 \\
\hline AA1 & A seems to be a bit over the area, but compared to B it's much better. & 6 & 3 & SaN_2 \\
\hline AA1 & A is perfect, covers the background a bit, but it's perfect. & 7 & 6 & SaN_2 \\
\hline SCC & $\begin{array}{l}\text { Robot A almost had a full match but also marked a little background. } \\
\text { Robot B had a full match but it also marked too much background. }\end{array}$ & 6 & 5 & SaN_2 \\
\hline XE2 & The $\mathrm{A}$ focuses on a single point of the figure but still is better than the $\mathrm{B}$ & 5 & 4 & SaN_1 \\
\hline XE2 & $\begin{array}{l}\text { Robot A was confident most of one feature of the glyph, while Robot B } \\
\text { misses most (not all) of the same feature. }\end{array}$ & 3 & 2 & SaN_2 \\
\hline ZC1 & The robot $\mathrm{B}$ emphasized more precise parts than the robot $\mathrm{A}$ & 3 & 6 & RN_1 \\
\hline ZU1 & $\begin{array}{l}\mathrm{B} \text { is slightly better as it covers more of the diagnostic areas whereas } \mathrm{A} \text { is } \\
\text { only about half of } \mathrm{B} \text {. }\end{array}$ & 3 & 5 & RN_1 \\
\hline ZU1 & B it's just a random mark in the middle, A seems to be more accurate. & 5 & 3 & SaN_1 \\
\hline ZU1 & $\begin{array}{l}\text { Robot A: Confident only at the edge of diagnostic part. Robot B: High- } \\
\text { lighted background and other parts mostly }\end{array}$ & 6 & 4 & SaN_2 \\
\hline $\begin{array}{l}\text { ZU1 (top in } \\
\text { Table 8) }\end{array}$ & $\begin{array}{l}\text { Robot A was almost perfect while the B one hardly touched the diagnostic } \\
\text { parts. }\end{array}$ & 6 & 4 & SaN_2 \\
\hline 1S2 & $\begin{array}{l}\text { Robot A had more than a half of the match and too much background. } \\
\text { Robot B had a little match and also matched a little background. }\end{array}$ & 5 & 4 & SaN_1 \\
\hline $\begin{array}{l}\text { 1G1 (top in } \\
\text { Table } 7 \text { ) }\end{array}$ & $\begin{array}{l}\text { Robot A missed one important part, and robot B marked big area of } \\
\text { unimportant part, so it is equal. }\end{array}$ & 5 & 5 & 0 \\
\hline $1 \mathrm{G} 1$ & $\begin{array}{l}\text { Robot B confident only at the edge of diagnostic part, Robot A missed } \\
\text { some of diagnostic part }\end{array}$ & 5 & 7 & RN_1 \\
\hline $1 \mathrm{G} 1$ & $\begin{array}{l}\text { Figure A, although not so much encompassing, focuses on the crucial } \\
\text { points of the form }\end{array}$ & 5 & 4 & SaN_1 \\
\hline 1B1 & $\begin{array}{l}\text { Robot B was confident about the two features of the glyph, while Robot } \\
\text { A was confident about only one. }\end{array}$ & 5 & 6 & RN_2 \\
\hline
\end{tabular}

pretrained networks, specifically Sketch-a-Net, VGG-16, and ResNet-50, finetuning the last blocks of these pretrained nets, and training the sequential and residual CNN variants from scratch (LeNet, SaN, R18, and R50).

As answers to RQ1 and RQ2, we showed that transfer learning via fine-tuning of the last convolutional layer improved the classification performances considerably, compared to evaluating the pretrained representations directly. Moreover, we observed that VGG-16 pretrained network is more robust than the recent R50 for assessing the data with different nature (as is the case of glyphs). That said, training a sequential sketch-specific network with few parameters from scratch with batch normalization, balanced oversampling, and dropout regularization outperformed the other training strategies and the recent residual models. Note that this model achieved over $70 \%$ average top- 1 , and over $85 \%$ average top- 5 accuracy in the 150 -class case. This finding is quite promising for all the other visual shape recognition tasks with limited amount of data.

Furthermore, to address RQ3, we visualized the discriminative parts of glyphs via guided gradient backpropagation and Grad-CAM methods, and showed that the trained model has a great potential as the discriminative parts of glyphs matched with the expert descriptions in a 5-glyph case study. To our knowledge, this is the first time that expert knowledge in ancient Maya epigraphy is reflected in a fully data-driven machine inference process, i.e. that does not use hand-crafted shape descriptors. Additionally, we showed the potential of the Grad-CAM method 


\section{How to Tell Ancient Signs Apart? \\ Recognizing and Visualizing Maya Glyphs with CNNs • 1:23}

in glyph localization in a cluttered setting, i.e. glyph-blocks. Finally, to address RQ4, we investigated on how to assess and exploit $\mathrm{CNN}$ visual outputs in a comparative study. According to this study, sketch-specific network $(\mathrm{SaN})$ visualizations were found more focused and appealing compared to the more-diffused visualizations of the residual network (ResNet-50). The ResNet-50 visualizations were favored only in the case of the glyph categories that require a large region to diagnose. Therefore, we conclude that overall the crowdworkers perceived the SaN visualizations more precise and insightful than the ResNet-50 visualizations in order to locate the diagnostic parts of the glyphs.

\section{ACKNOWLEDGMENTS}

This work was funded by the Swiss National Science Foundation through the MAAYA project and by the Hasler Foundation through the DCrowdLens project. We thank Carlos Pallán Gayol (Univ. of Bonn), Guido Krempel (Univ. of Bonn), Jacub Spotak (Comenius Univ. in Bratislava) for generating the glyph block dataset and for providing the glyph annotations, and Rui Hu (Idiap) for discussions. The original images that are adapted in the figures and the tables were created by Carlos Pallán Gayol for the MAAYA project.

\section{REFERENCES}

[1] Abrar H Abdulnabi, Gang Wang, Jiwen Lu, and Kui Jia. 2015. Multi-task CNN model for attribute prediction. IEEE Transactions on Multimedia 17, 11 (2015), 1949-1959.

[2] Gulcan Can, Jean-Marc Odobez, and Daniel Gatica-Perez. 2016. Evaluating Shape Representations for Maya Glyph Classification. ACM Journal on Computing and Cultural Heritage (JOCCH) 9, 3 (sep 2016).

[3] Gulcan Can, Jean-Marc Odobez, and Daniel Gatica-Perez. 2017. Maya Codical Glyph Segmentation: A Crowdsourcing Approach. Transactions on Multimedia (in press) (September 2017). https://doi.org/10.1109/TMM.2017.2755985

[4] Gülcan Can, Jean-Marc Odobez, and Daniel Gatica-Perez. 2017. Shape Representations for Maya Codical Glyphs: Knowledge-driven or Deep?. In Proceedings of the 15th International Workshop on Content-Based Multimedia Indexing (CBMI '17). ACM, Article 32, 6 pages. https://doi.org/10.1145/3095713.3095746

[5] Alfredo Canziani, Adam Paszke, and Eugenio Culurciello. 2016. An Analysis of Deep Neural Network Models for Practical Applications. arXiv preprint arXiv:1605.07678 (2016).

[6] Dan C Cireşan, Ueli Meier, and Jürgen Schmidhuber. 2012. Transfer learning for Latin and Chinese characters with deep neural networks. In Neural Networks (IJCNN), The 2012 International Joint Conference on. IEEE, 1-6.

[7] Jia Deng, Wei Dong, Richard Socher, Li-Jia Li, Kai Li, and Li Fei-Fei. 2009. Imagenet: A large-scale hierarchical image database. In Computer Vision and Pattern Recognition. IEEE, 248-255.

[8] V. Deufemia, L. Paolino, and H. d. Lumley. 2012. Petroglyph Recognition Using Self-Organizing Maps and Fuzzy Visual Language Parsing. In IEEE 24th International Conference on Tools with Artificial Intelligence, Vol. 1. 852-859. https://doi.org/10.1109/ICTAI.2012.119

[9] Jeff Donahue, Yangqing Jia, Oriol Vinyals, Judy Hoffman, Ning Zhang, Eric Tzeng, and Trevor Darrell. 2014. DeCAF: A Deep Convolutional Activation Feature for Generic Visual Recognition.. In ICML, Vol. 32. 647-655.

[10] Mathias Eitz, James Hays, and Marc Alexa. 2012. How Do Humans Sketch Objects? ACM Trans. Graph. 31, 4, Article 44 (jul 2012), 10 pages.

[11] Morris Franken and Jan C van Gemert. 2013. Automatic egyptian hieroglyph recognition by retrieving images as texts. In ACM Multimedia Conference. ACM, 765-768.

[12] Xavier Glorot and Yoshua Bengio. 2010. Understanding the difficulty of training deep feedforward neural networks. In International Conference on Artificial Intelligence and Statistics. 249-256.

[13] Kaiming He, Xiangyu Zhang, Shaoqing Ren, and Jian Sun. 2016. Deep residual learning for image recognition. In Proceedings of the IEEE Conference on Computer Vision and Pattern Recognition. 770-778.

[14] Paulina Hensman and David Masko. 2015. The Impact of Imbalanced Training Data for Convolutional Neural Networks. Technical Report. KTH, Stockholm, Sweden. Degree Project, in Computer Science, First Level.

[15] Geoffrey E Hinton, Nitish Srivastava, Alex Krizhevsky, Ilya Sutskever, and Ruslan R Salakhutdinov. 2012. Improving neural networks by preventing co-adaptation of feature detectors. arXiv preprint arXiv:1207.0580 (2012).

[16] Judy Hoffman, Eric Tzeng, Jeff Donahue, Yangqing Jia, Kate Saenko, and Trevor Darrell. 2013. One-shot adaptation of supervised deep convolutional models. arXiv preprint arXiv:1312.6204 (2013).

[17] Stephen Houston, John Robertson, and David Stuart. 2000. The Language of Classic Maya Inscriptions. Current Anthropology 41, 3 (2000), 321-356. https://doi.org/10.1086/300142 
[18] Rui Hu, Gulcan Can, Carlos Pallan Gayol, Guido Krempel, Jakub Spotak, Gabrielle Vail, Stephane Marchand-Maillet, Jean-Marc Odobez, and Daniel Gatica-Perez. 2015. Multimedia Analysis and Access of Ancient Maya Epigraphy. Signal Processing Magazine 32, 4 (July 2015), 75-84.

[19] Sergey Ioffe and Christian Szegedy. 2015. Batch Normalization: Accelerating Deep Network Training by Reducing Internal Covariate Shift. In Proc. of International Conference on Machine Learning. 448-456.

[20] Diederik Kingma and Jimmy Ba. 2014. Adam: A method for stochastic optimization. arXiv preprint arXiv:1412.6980 (2014).

[21] Alex Krizhevsky, Ilya Sutskever, and Geoffrey E Hinton. 2012. Imagenet classification with deep convolutional neural networks. In Advances in Neural Information Processing Systems. 1097-1105.

[22] Yann LeCun, Yoshua Bengio, and Geoffrey Hinton. 2015. Deep learning. Nature 521, 7553 (2015), 436-444.

[23] Yann LeCun, Léon Bottou, Yoshua Bengio, and Patrick Haffner. 1998. Gradient-based learning applied to document recognition. Proc. IEEE 86, 11 (1998), 2278-2324.

[24] David G. Lowe. 1999. Object recognition from local scale-invariant features. In International Conference on Computer Vision, Vol. 2. IEEE, $1150-1157$.

[25] Martha J. Macri and Matthew George Looper. 2003. The New Catalog of Maya Hieroglyphs: The Classic Period Inscriptions. Vol. 1. University of Oklahoma Press.

[26] Martha J. Macri and Gabrielle Vail. 2008. The New Catalog of Maya Hieroglyphs, vol. 2: The Codical Texts. University of Oklahoma Press.

[27] Frank J Massey Jr. 1951. The Kolmogorov-Smirnov test for goodness of fit. Journal of the American statistical Association 46, 253 (1951), 68-78.

[28] Georg Poier, Markus Seidl, Matthias Zeppelzauer, Christian Reinbacher, Martin Schaich, Giovanna Bellandi, Alberto Marretta, and Horst Bischof. 2017. The 3D-Pitoti Dataset: A Dataset for High-resolution 3D Surface Segmentation. In Proceedings of the 15th International Workshop on Content-Based Multimedia Indexing (CBMI '17). ACM, New York, NY, USA, Article 5, 7 pages. https: //doi.org/10.1145/3095713.3095719

[29] Edgar Roman-Rangel, Gulcan Can, Stephane Marchand-Maillet, Rui Hu, Carlos Pallan Gayol, Guido Krempel, Jakub Spotak, Jean-Marc Odobez, and Daniel Gatica-Perez. 2016. Transferring Neural Representations for Low-dimensional Indexing of Maya Hieroglyphic Art. In ECCV Workshop on Computer Vision for Art Analysis.

[30] Edgar Roman-Rangel, Carlos Pallan, Jean-Marc Odobez, and Daniel Gatica-Perez. 2011. Analyzing ancient maya glyph collections with contextual shape descriptors. IJCV 94, 1 (2011), 101-117.

[31] Markus Seidl and Christian Breiteneder. 2012. Automated Petroglyph Image Segmentation with Interactive Classifier Fusion. In Proceedings of the Eighth Indian Conference on Computer Vision, Graphics and Image Processing (ICVGIP '12). ACM, New York, NY, USA, Article 66, 8 pages. https://doi.org/10.1145/2425333.2425399

[32] Markus Seidl, Ewald Wieser, and Craig Alexander. 2015. Automated classification of petroglyphs. Digital Applications in Archaeology and Cultural Heritage 2, 2 (2015), 196 - 212. https://doi.org/10.1016/j.daach.2015.03.001 Digital imaging techniques for the study of prehistoric rock art.

[33] Markus Seidl, Ewald Wieser, Matthias Zeppelzauer, Axel Pinz, and Christian Breiteneder. 2015. Graph-Based Shape Similarity of Petroglyphs. Springer International Publishing, Cham, 133-148. https://doi.org/10.1007/978-3-319-16178-5_9

[34] Ramprasaath R Selvaraju, Abhishek Das, Ramakrishna Vedantam, Michael Cogswell, Devi Parikh, and Dhruv Batra. 2016. Grad-CAM: Why did you say that? arXiv preprint arXiv:1611.07450 (2016).

[35] Ali Sharif Razavian, Hossein Azizpour, Josephine Sullivan, and Stefan Carlsson. 2014. CNN Features Off-the-Shelf: An Astounding Baseline for Recognition. In CVPR Workshops.

[36] Karen Simonyan, Andrea Vedaldi, and Andrew Zisserman. 2013. Deep inside convolutional networks: Visualising image classification models and saliency maps. arXiv preprint arXiv:1312.6034 (2013).

[37] K. Simonyan and A. Zisserman. 2014. Very Deep Convolutional Networks for Large-Scale Image Recognition. CoRR abs/1409.1556 (2014).

[38] Christian Szegedy, Sergey Ioffe, Vincent Vanhoucke, and Alex Alemi. 2016. Inception-v4, inception-resnet and the impact of residual connections on learning. arXiv preprint arXiv:1602.07261 (2016).

[39] Christian Szegedy, Wei Liu, Yangqing Jia, Pierre Sermanet, Scott Reed, Dragomir Anguelov, Dumitru Erhan, Vincent Vanhoucke, and Andrew Rabinovich. 2015. Going deeper with convolutions. In Proceedings of the IEEE Conference on Computer Vision and Pattern Recognition. 1-9.

[40] N. Tajbakhsh, J. Y. Shin, S. R. Gurudu, R. T. Hurst, C. B. Kendall, M. B. Gotway, and J. Liang. 2016. Convolutional Neural Networks for Medical Image Analysis: Full Training or Fine Tuning? IEEE Transactions on Medical Imaging 35, 5 (May 2016), 1299-1312.

[41] John Eric Sidney Thompson and George E. Stuart. 1962. A Catalog of Maya Hieroglyphs. University of Oklahoma Press.

[42] Jason Yosinski, Jeff Clune, Yoshua Bengio, and Hod Lipson. 2014. How transferable are features in deep neural networks?. In Advances in Neural Information Processing Systems. 3320-3328. 
How to Tell Ancient Signs Apart?

Recognizing and Visualizing Maya Glyphs with CNNs • 1:25

[43] Qian Yu, Yongxin Yang, Yi-Zhe Song, Tao Xiang, and Timothy Hospedales. 2015. Sketch-a-net that beats humans. arXiv preprint arXiv:1501.07873 (2015).

[44] Matthew D Zeiler and Rob Fergus. 2014. Visualizing and understanding convolutional networks. In European conference on computer vision. Springer, 818-833.

[45] B. Zhou, A. Khosla, Lapedriza. A., A. Oliva, and A. Torralba. 2016. Learning Deep Features for Discriminative Localization. CVPR (2016).

[46] Qiang Zhu, Xiaoyue Wang, Eamonn Keogh, and Sang-Hee Lee. 2011. An efficient and effective similarity measure to enable data mining of petroglyphs. Data Mining and Knowledge Discovery 23, 1 (01 Jul 2011), 91-127. https://doi.org/10.1007/s10618-010-0200-z

Received September 2017; revised November 2017; revised May 2018; accepted June 2018 